\title{
Hamilton in Brüssel? Europa- und verfassungsrechtliche Aspekte der Reform des EU-Eigenmittelsystems und des Next Generation-Programms der E $\mathbf{U}^{*}$
}

\begin{abstract}
The new Multiannual Financial Framework (MFF) and the decisions on the EU'S long-term budget have enabled the European Union to regain visibility in the Covid crisis thanks to its Next Generation EU (NGEU) approach, which includes $€ 750$ billion in financial support and the introduction of new EU borrowing. This article analyzes the European and German constitutional law implications of the economic aid measures agreed in the NGEU context. These measures are based on Articles 122 and 311 TFEU, but the legal basis is not uncontested. According to Article 311 TFEU, ratification in the Member States is necessary, which implies a Member State constitutional law dimension of NGEU. In Germany, the constitutional law debate concerned the question of whether ratification should be carried out by Parliament by a simple majority or a two-thirds majority. The overall assessment is that although NGEU is a major step, it is not a constitutional or a Hamiltonian moment.
\end{abstract}

\section{Résumé}

Le nouveau cadre financier pluriannuel (CFP) et les décisions sur le budget à long terme de l'UE ont permis à l'Union européenne a retrouvé son rôle dans la pandémie grâce à son approche "Next Generation EU (NGEU) qui porte entre autres sur 750 milliards d'euros d'aide financière et la mise en place, nouvelle, d'emprunts de l'UE. Cet article analyse les enjeux de droit européen et de droit constitutionnel allemand des mesures d'aide économique convenues dans le cadre de NGEU. Ces mesures sont fondées sur les articles 122 et 311 du TFUE, mais cette base juridique est contestée. Selon l'article 311 du TFUE, une ratification dans les États membres est nécessaire, ce qui implique une dimension de droit constitutionnel national de NGEU. En Allemagne, le débat de droit constitutionnel a porté sur la question de savoir si la ratification doit être effectuée par le Parlement à la majorité simple ou à la majorité des deux tiers. La vue globale du sujet est que bien que NGEU soit une étape majeure, il ne s'agit pas d'un moment constitutionnel ou " hamiltonien ».

* Prof. Dr. Franz C. Mayer, LL.M. (Yale), ist Inhaber des Lehrstuhls für Öffentliches Recht, Europarecht, Völkerrecht, Rechtsvergleichung und Rechtspolitik an der Fakultät für Rechtswissenschaft, Universität Bielefeld. Philipp Lütkemeyer ist dort Wiss. Mitarbeiter. Der Beitrag entwickelt eine Stellungnahme für eine Sachverständigenanhörung zu „Next Generation EU“ im Europaausschuss des Deutschen Bundestags vom Oktober 2020 weiter. Erst nach Einreichung des Manuskripts wurde Prof. Dr. Franz C. Mayer zum Prozessbevollmächtigten des Deutschen Bundestages im NGEU betreffenden Verfahren vor dem BVerfG (2 BvR 547/21) bestellt. Entsprechend stehen die nachfolgenden Überlegungen in keinem Zusammenhang zu diesem Mandat. 


\section{Hintergrund}

Öffentliche Einnahmen, wer sie beschließt, wie sie begründet und wie sie legitimiert werden, dies gehört zu den ältesten Verfassungsthemen überhaupt. „Power of the purse“, „Taxation and representation“ und das „Königsrecht der Parlamente“ sind nur einige Stichworte, die dies illustrieren. Auch im Kontext der europäischen Integration stellt sich dieses Verfassungsthema. Je weiter die Europäische Union sich von herkömmlichen Internationalen Organisationen entfernt, desto deutlicher treten Grundfragen der Verfassungstheorie hervor, die sich für Beitragszahlungen Deutschlands an internationale Organisationen in aller Regel nicht stellen. Dass sich nirgendwo sonst im überstaatlichen Raum eine mit dem direkt gewählten Europäischen Parlament vergleichbare parlamentarische Komponente findet, ist nur eine der zahlreichen Besonderheiten der europäischen Konstellation. Zugleich ist aber auch leicht erkennbar, dass die Europäische Union noch immer weit weg davon ist, sich in Sachen Einnahmen zu verselbständigen. Eine nennenswerte eigene Unionssteuer ist nicht in Sicht. Bis auf Weiteres lässt sich der Satz „no taxation without representation“ nicht umkehren in „no representation without taxation“: Das Europäische Parlament verfügt gerade nicht über die Haushaltsautonomie, sondern wirkt mit anderen Akteuren in Haushaltsfragen zusammen. Die Mitgliedstaaten kontrollieren über die Mittelausstattung der Europäischen Union letztlich die Reichweite europäischer Politik und eine Verselbständigung der europäischen Ebene, was je nach Standpunkt als Problembeschreibung oder als Konzeptmerkmal gedeutet werden wird. Gleichwohl belegt das Beispiel NGEU sehr deutlich, dass die Verträge durchaus weitreichende Kompetenzen im Hinblick auf Einnahmen zugunsten der Union vorsehen, so denn die politischen Mehrheiten bestehen.

\section{Die Pandemie als Katalysator?}

In der Pandemie-Krise scheinen sich mit der Reform des EU-Eigenmittelsystems vom Dezember 2020 die Dinge in Bewegung zu setzen. Es gehört dabei sicherlich zu den positiven Aspekten der Entwicklung, dass die EU so als Akteur in der Krise wieder sichtbar wird, nachdem zu Beginn mit hektischen Grenzschließungen ein Rückzug auf nationalstaatliche Verhaltensmuster erfolgte, der eher reflexhaft als überlegt daherkam. Dass die EU in Sachen Gesundheitspolitik von den Mitgliedstaaten absichtsvoll mit wenigen und schwachen Kompetenzen ausgestattet ist, ist auch Teil der Erklärung für die anfängliche Abwesenheit der EU in der Krise. Für die Bewältigung der ökonomischen Folgen ist die EU, deren zentrales Leistungsmerkmal nach wie vor der Binnenmarkt in Europa ist, hingegen ein ganz naheliegender Akteur. Dabei ist es letztlich dem Zufall geschuldet, dass ausgerechnet mitten in der Pandemie die Entscheidung über den nächsten mehrjährigen Finanzrahmen 2021-2027 zu treffen war.

Der Weg zu einer gemeinsamen Anstrengung um die ökonomischen Folgen der Pandemie zu bewältigen verlief dabei alles andere als gerade. ${ }^{1}$ Nach den kontroversen

$1 \mathrm{Zu}$ den Hintergründen $\mathrm{s}$. den Beitrag „The coronavirus recovery plan that von der Leyen built“" v. 15.7.2020, https://www.politico.eu. 
Diskussionen um Corona-Bonds und einem ergebnislos verlaufenen Europäischen Rat im März 2020 wurde die Grundkonzeption des Maßnahmenpakets „Next Generation EU“ (NGEU) Ende Mai 2020 durch die Europäische Kommission vorgeschlagen, ${ }^{2}$ nachdem zuvor die gemeinsame Initiative des französischen Staatspräsidenten Macron und der deutschen Bundeskanzlerin Merkel $^{\beta}$ einen Durchbruch gebracht hatte.

Im Sommer 2020 gelang dann die grundsätzliche Einigung aller Staats- und Regierungschefs bei der Außerordentlichen Tagung des Europäischen Rates vom 17.-21. Juli 2020. ${ }^{4}$ Die Umsetzung dieser Vorgabe erfolgte mit der Annahme eines Beschlusses des Rates über das System der Eigenmittel der Europäischen Union am 14. Dezember $2020^{5}$ und der Verständigung auf einen neuen Mehrjährigen Finanzrahmen (MFR 2021-2027). Neben brexitbedingten Anpassungen des Eigenmittelbeschlusses und der Einführung einer neuen Eigenmittelkategorie („Plastik-Abgabe“) wird die Europäische Kommission im neuen Eigenmittelbeschluss ermächtigt, außerordentliche und zeitlich befristete zusätzliche Mittel zur Bewältigung der Folgen der COVID-19-Krise an den Kapitalmärkten im Namen der Union Mittel bis zu 750 Mrd. $€$ aufzunehmen. ${ }^{6}$ Festgelegt ist ferner, dass von den aufgenommenen Mitteln bis zu 360 Mrd.€ für die Gewährung von Darlehen und bis zu 390 Mrd. € für Ausgaben verwendet werden können. Die Rückzahlung der Kredite, die zur Finanzierung von Ausgaben aufgenommen wurden, erfolgt aus dem EU-Haushalt. ${ }^{7}$

Die Einzelheiten des Maßnahmenpakets sind komplex. Mehrere Rechtsakte greifen ineinander. Im Dezember 2020 wurde neben dem (neuen) Eigenmittelbeschluss mit einer auf Art. 122 AEUV gestützten Verordnung das „Aufbauinstrument“ der Europäischen Union zur Unterstützung der Erholung nach der COVID-19-Krise geschaffen. ${ }^{8}$

2 Mitteilung der Kommission, „Die Stunde Europas - Schäden beheben und Perspektiven für die nächste Generation eröffnen“, $\operatorname{COM(2020)} 456$ final v. 27.5.2020; Mitteilung der Kommission, „Der EU-Haushalt als Motor für den Europäischen Aufbauplan“, COM(2020) 442 final v. 27.5.2020; flankiert durch Sekundärrechtsentwürfe: Vorschlag für eine Verordnung des Rates zur Schaffung eines Aufbauinstruments der Europäischen Union zur Unterstützung der Erholung nach der COVID-19-Pandemie, $\operatorname{COM}(2020) 441$ final v. 28.5.2020 und einen Vorschlag für eine Verordnung des Europäischen Parlaments und des Rates zur Einrichtung einer Aufbau- und Resilienzfazilität, $\operatorname{COM(2020)} 408$ final v. 28.5.2020.

3 Deutsch-französische Initiative zur wirtschaftlichen Erholung Europas nach der Coronakrise, Pressemitteilung der Bundesregierung Nr. 173/2020 v. 18.5.2020.

4 Schlussfolgerungen des Europäischen Rates, Außerordentliche Tagung v. 17., 18., 19., 20. und 21.7.2020, EUCO 10/20 v. 21.7.2020.

5 Beschluss (EU, Euratom) 2020/2053 des Rates v. 14.12.2020 über das Eigenmittelsystem der Europäischen Union und zur Aufhebung des Beschlusses 2014/335/EU, Euratom, AB1. L 424 v. 15.12.2020, 1 (im Folgenden Eigenmittelbeschluss).

6 Art. 5 Eigenmittelbeschluss.

7 S. im Einzelnen BT-Drs. 19/26821 v. 19.2.2021, Entwurf eines Gesetzes zum Beschluss des Rates vom 14. Dezember 2020 über das Eigenmittelsystem der Europäischen Union und zur Aufhebung des Beschlusses 2014/335/EU, Euratom (Eigenmittelbeschluss-Ratifizierungsgesetz - ERatG).

8 Verordnung (EU) 2020/2094 des Rates vom 14. Dezember 2020 zur Schaffung eines Aufbauinstruments der Europäischen Union zur Unterstützung der Erholung nach der COVID-19-Krise (im Folgenden Aufbauinstrument-VO), ABl. L 433 I v. 15.12.2020, 23. 
Das Instrument wird auf der Grundlage des Eigenmittelbeschlusses bis in Höhe des Betrags von $750 \mathrm{Mrd}$ € finanziert.

Näher ausgestaltet und zugleich gebunden wird die Mittelverwendung im Wesentlichen ${ }^{9}$ durch die auf einer Verordnung vom Februar $2021^{10}$ beruhende „Aufbau- und Resilienzfazilität" in Höhe von 672,5 Mrd. €. Um daraus Unterstützung zu erhalten, müssen die Mitgliedstaaten nationale Aufbau- und Resilienzpläne mit Reform- und Investitionsplänen bis 2026 vorlegen. Dabei müssen sechs Politikbereiche von europäischer Tragweite abgedeckt sein: ökologischer Wandel; digitaler Wandel; intelligentes, nachhaltiges und integratives Wachstum und Beschäftigung; sozialer und territorialer Zusammenhalt; Gesundheit und Resilienz; Maßnahmen für die nächste Generation, Kinder und Jugendliche, einschließlich für Bildung und Kompetenzen. Ferner wird die Unterstützung mit den länderspezifischen Empfehlungen im Rahmen des Europäischen Semesters verknüpft.

Vorgesehen war, dass die Mitgliedstaaten bis zum 30. April 2021 der Kommission ihre Aufbau- und Resilienzpläne vorlegen. ${ }^{11}$ Nach Bewertung durch die Europäische Kommission entscheidet der Rat über die Genehmigung des jeweiligen Plans. Mittel werden nach Maßgabe der Erreichung der vereinbarten Etappenziele und Zielwerte ausgezahlt.

Notwendige Bedingung ist indessen, dass der (neue) Eigenmittelbeschluss der EU vom Dezember 2020 in allen Mitgliedstaaten ratifiziert ist, da die Kommission durch diesen Beschluss erst ermächtigt wird, die besagten Mittel zur Bewältigung der Folgen der COVID-19-Krise auf den Kapitalmärkten aufzunehmen.

Die für das Inkrafttreten des neuen Eigenmittelbeschlusses erforderlichen verfassungsrechtlichen Zustimmungsverfahren in den Mitgliedstaaten (vgl. Art. 311 AEUV) sind Anfang 2021 angelaufen. In Deutschland ist ein auf Art. 23 GG gestütztes Gesetz zum Beschluss des Rates vom 14. Dezember 2020 über das Eigenmittelsystem der Europäischen Union nach einem erfolglosen Eilverfahren vor dem BVerfG am 23. April 2021 in Kraft getreten. ${ }^{12}$

9 77,55 Mrd. $€$ werden über bestehende EU-Programme ausgereicht.

10 Verordnung (EU) 2021/241 des Europäischen Parlaments und des Rates vom 12. Februar 2021 zur Einrichtung der Aufbau- und Resilienzfazilität (im Folgenden AufbauinstrumentDurchführungs-VO), AB1. L 57 v. 18.2.2021, 17.

11 Die Bundesregierung hat bereits am 16. Dezember 2020 den Entwurf des Deutschen Aufbau- und Resilienzplans (DARP) im Bundeskabinett beschlossen und diesen der Europäischen Kommission zur Prüfung vorgelegt. Die Verhandlungen über den endgültigen DARP mit der Europäischen Kommission sollten bis Ende April 2021 abgeschlossen sein.

12 S. zu den Einzelheiten BVerfG, Beschl. v. 15.4.2021, 2 BvR 547/21 (Eilrechtsschutz). Das Gesetzesvorhaben wurde noch im Dezember 2020 im Bundesrat auf den Weg gebracht, s. Entwurf eines Gesetzes zum Beschluss des Rates vom 14. Dezember 2020 über das Eigenmittelsystem der Europäischen Union und zur Aufhebung des Beschlusses 2014/335/EU, Euratom (Eigenmittelbeschluss-Ratifizierungsgesetz - ERatG), Bundesrat-Drs. 764/20 v. 17.12.2020, s. auch BT-Drs. 19/26821 v. 19.2.2021. In 10 Mitgliedstaaten ist eine parlamentarische Zustimmungsgesetzgebung erforderlich. Bis zur Drucklegung dieses Beitrages hatten 19 Mitgliedstaaten die Ratifikation abgeschlossen. 


\section{Ein Hamilton-Moment?}

Über die politische Einordnung dieser Entwicklung besteht Uneinigkeit. Die weitreichendste Deutung rückt sie in die Nähe eines „Hamiltonschen Moments“, einer für die amerikanische föderale Entwicklung als maßgeblich angesehenen Schuldenübernahme durch den Bund. ${ }^{13}$ Die Gegenposition kritisiert diese Bezugnahme und bestreitet bereits, dass das Maßnahmenpaket sich im bestehenden Rechtsrahmen hält, ${ }^{14}$ meist unter mehr oder weniger schematischer Wiederholung der Einwände aus der Eurokrise. So wird im Hinblick auf die Verschuldensmöglichkeit der EU bezweifelt, dass die Gründungsverträge ausreichende Kompetenzgrundlagen aufweisen.

Bei genauerem Hinsehen erweist sich eine vermittelnde Position als am plausibelsten. Auch wenn die Maßnahmen ohne Präzedenz in der EU sind, so bedeutet dies nicht zugleich eine qualitative Verschiebung in Richtung Bundesstaatlichkeit. Der konkrete Kern der Maßnahmen bleibt zum übergroßen Anteil in nationaler Eigenverantwortung, weil die Mitgliedstaaten über ihre jeweiligen Aufbau- und Resilienzpläne im Rahmen der europäischen Vorgaben entscheiden. Ein Umbau der europäischen Finanzarchitektur erfolgt gerade nicht. Das Maßnahmenpaket ist ein großer Schritt, dies aber vor allem als politische Solidaritäts- und Einigungsleistung - rechtlich ist die nüchterne Feststellung, dass Art. 311 und 122 AEUV als Kompetenzgrundlagen tragen. Dies veranschaulicht zugleich, dass das Primärrecht bereits hinreichende Krisenbewältigungs- und Weiterentwicklungspotenziale bereit hält - wenn entsprechende politische Mehrheiten bestehen (dazu II.).

Das Primärrecht verweist im vorliegenden Kontext auf die nach dem jeweiligen Verfassungsrecht erforderlichen Maßnahmen in den Mitgliedstaaten. Auch hier lässt sich feststellen, dass die bestehenden Mechanismen bereits tragen und kein Zustimmungsgesetz nach Art. 23 GG mit verfassungsändernder Mehrheit erforderlich ist. Es genügt die einfache Mehrheit. Am Beispiel NGEU lassen sich zugleich Leistungsgrenzen eines Systemverständnisses, das auf Zweidrittelmehrheiten setzt, veranschaulichen (dazu III.).

\section{Unionsrechtliche Kompetenzgrundlagen}

\section{Einige Vorüberlegungen und Unterscheidungen}

Die der Europäischen Kommission übertragene Befugnis zur Mittelaufnahme (,Verschuldung") hat erhebliches Aufsehen erregt. Diese Mittelaufnahme ist im neuen Eigenmittelbeschluss hinsichtlich ihrer Höhe, der Dauer und ihres Zwecks klar begrenzt. Die generierten Mittel werden über die Instrumente und Programme des MFR für Ausgaben verwendet sowie als Darlehen an die Mitgliedstaaten vergeben - allerdings nur zur Verwendung im Rahmen des Aufbauinstrumentes NGEU zur Bewältigung der Folgen der COVID-19 Krise. Die Rückzahlung der Kredite, die zur Finanzierung von Ausgaben aufgenommen wurden, erfolgt aus dem EU-Haushalt.

13 S. in diesem Zusammenhang das Interview mit Bundesfinanzminister Scholz, „Jemand muss vorangehen“, in: DIE ZEIT Nr. 22/2020 v. 20.5.2020.

14 Statt aller Sinn, FAZ v. 22.5.2020, S. 17; Herdegen, FAZ v. 25.3.2021, S. 6. 
Für die Antwort auf die Frage nach der Kompetenzgrundlage für diese Konstruktion und insbesondere für die in NGEU vorgesehene Verschuldungsmöglichkeit der Union sind zunächst einige grundlegende Vorüberlegungen und Unterscheidungen hilfreich. So ist einerseits zwischen den Ebenen der Kapitalaufnahme, -verwendung und -rückführung sowie andererseits auf Ebene der Kapitalverwendung zwischen den konkreten Unterstützungen zu differenzieren.

1. Die Ebene der Kapitalaufnahme umfasst die Verschuldungsmöglichkeit der Union im Verhältnis zum Kapitalmarkt und gewährleistet die Ausstattung mit den notwendigen finanziellen Mitteln zur Finanzierung der mit NGEU adressierten Programme. Dazu wird die EU in Art. 5 Abs. 1 lit. a) Eigenmittelbeschluss ermächtigt.

2. Die Ebene der Kapitalverwendung regelt die konkreten Verwendungsmöglichkeiten des aufgenommenen Kapitals. Vorgesehen werden in der AufbauinstrumentVO nicht rückzahlbare und rückzahlbare Unterstützungen nach Art. 2 Abs. 2 lit. a), die Begabe von Darlehen zugunsten der Mitgliedstaaten, Art. 2 Abs. 2 lit. b), und die Dotierung von Garantien zur Unterstützung weiterer Programme, Art. 2 Abs. 2 lit. c).

3. Die Ebene der Kapitalrückführung bezieht sich schließlich auf diejenigen Regelungen, welche die Modalitäten und das Verfahren der Rückführung des geliehenen Kapitals nebst Bedienung etwaiger Zinsen betreffen. Kompetentiell ist die Kapitalrückführung spiegelbildlich zur Kapitalaufnahme konzipiert und ist ebenfalls im Eigenmittelbeschluss verankert, Art. 5 Abs. 2. Diese Ebene determiniert überdies die haushaltsrechtliche Dimension der NGEU: Art. 5 Abs. 2 UAbs. 1 S. 1 Eigenmittelbeschluss sieht vor, dass die Rückzahlung des Kapitalbetrags der aufgenommenen Mittel für Ausgaben und darauf fällig werdenden Zinsen zu Lasten des Unionshaushaltes gehen. Offenkundig spielen hier die nicht rückzahlbaren Unterstützungsleistungen eine entscheidende Rolle.

Als nächstes ist für die Beurteilung nachfolgende Unterscheidung zwischen nicht rückzahlbaren und rückzahlbaren Unterstützungen hilfreich:

- Rückzahlbare Unterstützungen sind solche Unterstützungen, die zwar eine Leistung seitens der Union zugunsten des Mitgliedstaats umfassen, jedoch einen Rückzahlungsanspruch in selbiger Höhe nebst ggf. Zinszahlungen begründen. Hier ist insbesondere an das Ausreichen von Darlehen ,zur Finanzierung des Aufbaus und der wirtschaftlichen und sozialen Resilienz durch die Unterstützung von Reformen und Investitionen“ gem. Art. 2 Abs. 2 lit.b) Aufbauinstrument-VO zu denken. Aufgrund des verbrieften Rückzahlungsanspruchs sind solche Maßnahmen grundsätzlich haushaltsneutral, solange die ausgegebenen Mittel im Verhältnis Union zu Mitgliedstaat vollständig zurückgeführt und die der Union entstehenden Zinskosten an die Mitgliedstaaten weitergereicht werden. Mithin entsteht eine haushaltswirksame (Re-)Finanzierungsnotwendigkeit dann, wenn Mitgliedstaaten die geliehenen Mittel nicht vollumfänglich zurückführen oder Zinszahlungen nicht leisten können oder die Union die Finanzmittel zu einem günstigeren Zins weitergibt, als sie selbst im Rahmen der Kapitalaufnahme am Markt bieten musste.

- Nicht rückzahlbare Unterstützungen (verlorene Zuschüsse) sind demgegenüber eine Unterstützungsform, welche durch den endgültigen Verbleib der Finanzmittel im Zielprogramm gekennzeichnet sind. Ihnen steht somit gerade kein Rückzah- 
lungsanspruch gegenüber. Solche Unterstützungen sind damit stets haushaltswirksam, indem die Rückzahlungspflicht der am Markt aufgenommen Finanzmittel vollständig durch den Unionshaushalt zu tragen ist, Art. 5 Abs. 2 Eigenmittelbeschluss. Mit einem Volumen von insgesamt 384,4 Mrd. $€$ nehmen die nicht rückzahlbaren Unterstützungen für Programme wie zur Finanzierung des Aufbaus der wirtschaftlichen und sozialen Resilienz durch die Unterstützung von Reformen und Investitionen, Struktur- und Kohäsionsprogramme, oder Programme zur Unterstützung von Gebieten bei ihrem Übergang zu einer klimaneutralen Wirtschaft, Art. 2 Abs. 2 lit. a) Aufbauinstrument-VO, den größten Teil der im Rahmen der durch NGEU konzipierten Programme ein. 5,6 Mrd. $€$ werden für die Dotierung für Haushaltsgarantien bereitgestellt. Insgesamt sollen damit $390 \mathrm{Mrd}$. $€$ als nicht rückzahlbare Unterstützungen freigemacht werden, Art. 5 Abs. 1 lit. b) Eigenmittelbeschluss. Daneben werden $360 \mathrm{Mrd}$. $€$ als rückzahlbare Unterstützungen für Darlehen an die Mitgliedstaaten bereitgestellt, Art. 2 Abs. 2 lit. b) Aufbauinstrument-VO.

\section{Kompetenzgrundlagen von NGEU: Art. 122 und Art. 311 AEUV}

Bezüglich der relevanten Kompetenzgrundlagen ist zwischen den soeben eingeführten Ebenen zu unterscheiden. Die Kapitalaufnahme (Art. 5 Abs. 1 Eigenmittelbeschluss) sowie die Kapitalrückführung (Art. 5 Abs. 2 Eigenmittelbeschluss) werden auf Art. 311 Abs. 3 AEUV und damit auf den Eigenmittelbeschluss, die Kapitalverwendung (Art. 2 Aufbauinstrument-VO) hingegen auf Art. 122 AEUV gestützt. ${ }^{15}$

Diese Kompetenzspreizung auf Art. 311 respektive Art. 122 AEUV erstaunt vielleicht vor dem Hintergrund historischer Beispiele, in denen sich die Union oder die Gemeinschaft der Aufnahme von Fremdkapital bedienten. Bisher wurde bei schuldenfinanziertem Beistand der EU sowohl die Kapitalverwendung wie auch die Kapitalaufnahme und -rückführung des finanziellen Beistands ausschließlich auf die materiell-rechtliche Durchführungskompetenz wie etwa Art.122 Abs. 2 AEUV für den EFSM (bzw. auf Art. 143 AEUV bei Zahlungsbilanzhilfen) gestützt. ${ }^{16}$

Damit stellt sich die Frage, warum nun Art.311 AEUV in Verbindung mit Art. 122 AEUV als Kompetenzgrundlage gewählt wird bzw. weshalb es des Rückgriffs auf die haushaltrechtlichen Bestimmungen bedarf.

$15 \mathrm{Zu}$ kurz daher Herdegen, der auch die Kapitalaufnahme auf Art. 122 AEUV gestützt sieht: „Die Beistandsklausel im Artikel 122 des EU-Vertrages ist keine Grundlage dafür, dass sich die EU Geld außerhalb des Haushalts besorgen darf.“, Scheitert der Wiederaufbaufonds?, Handelsblatt v. 22.3.2021, S. 9.

16 Siehe beispielsweise die EFSM-VO, Verordnung (EU) Nr. 407/2010 des Rates vom 11. Mai 2010, die nur auf Art. 122 Abs. 2 AEUV gestützt wurde; ebenso im Fall der Zahlungsbilanzanleihen („,Petro-Dollar-Anleihen“) zur Bekämpfung der Folgen der ersten Ölkrise in den 70er Jahre, welche nur auf Art. 308 EGV, jetzt Art. 352 AEUV, gestützt wurde. 


\section{a) Kapitalaufnahme, Art. 5 Abs. 1 Eigenmittelbeschluss}

Für die Antwort auf diese Frage ist es hilfreich, sich zunächst über die Besonderheiten der Mittel, um die es vorliegend geht, zu vergewissern. Als Kompetenznorm kommt in dem Kontext konkret Art. 311 Abs. 3 AEUV in Frage. Dieser regelt das System der Eigenmittel der Union. Er lautet:

„Der Rat erlässt gemäß einem besonderen Gesetzgebungsverfahren einstimmig und nach Anhörung des Europäischen Parlaments einen Beschluss, mit dem die Bestimmungen über das System der Eigenmittel der Union festgelegt werden. Darin können neue Kategorien von Eigenmitteln eingeführt oder bestehende Kategorien abgeschafft werden. Dieser Beschluss tritt erst nach Zustimmung der Mitgliedstaaten im Einklang mit ihren jeweiligen verfassungsrechtlichen Vorschriften in Kraft. "

Der Begriff der Eigenmittel geht auf Art. 201 EWGV zurück. Er löste die in Art. 200 EWGV geregelten „Finanzbeiträge“ der Mitgliedstaaten ab und stellt seit dem Vertrag von Maastricht die wichtigste Finanzierungsquelle der Union dar. ${ }^{17}$ Ziel dieser Umstellung war seinerzeit, die Europäische Gemeinschaft mit einer größeren Finanzautonomie auszustatten, als es für von Beiträge ihrer Mitglieder abhängige internationale Organisationen typisch war und auch noch heute ist. ${ }^{18}$ Bezweckt wurde damit ein höherer Grad an Unabhängigkeit der Gemeinschaft gegenüber der Zahlungsbereitschaft ihrer Mitgliedstaaten. Eigenmittel sollten der Gemeinschaft deshalb „quasi automatisch bereitgestellt werden“19 ohne erst durch den nationalen Haushalt bewilligt werden zu müssen. Eigenmittel verbinden sich demnach historisch zuvörderst mit dem Gedanken der unionsautonomen Finanzierung.

Dazu sieht Art. 311 Abs. 3 AEUV vor, dass sich die Union mit den Mitgliedstaaten zusammen ein System von Eigenmitteln gibt, welches die Abschaffung alter und die Einführung neuer Kategorien von Eigenmitteln umfasst. Im Schrifttum herrscht zur Reichweite des Systems von Eigenmitteln Uneinigkeit, ob und inwieweit der Union aus anderen Normen des AEUV $^{20}$ materiell-rechtliche Einschränkungen für das System von Eigenmitteln vorgegeben werden ${ }^{21}$ oder ob es lediglich auf ein formelles Begriffsverständnis der Eigenmittel derart ankommt, dass Eigenmittel sind, was in dem dafür vorgesehenen Verfahren als Eigenmittel eingeführt wurden. ${ }^{22}$ Eine mögliche Konsequenz einer der Ausgangspositionen ist die Annahme, dass die Aufnahme von Finanzmitteln am Markt nicht vom System der Eigenmittel umfasst werden darf, je-

17 S. Magiera, in: Grabitz/Hilf/Nettesheim, Recht der EU, Art. 311 AEUV, Rn. 1.

18 Als prominentes und aktuelles Beispiel für die Problematik der Abhängigkeit internationaler Organisationen von den Mitgliedsbeiträgen ihrer Mitglieder mag das Beispiel der WHO dienen, deren finanzielle Unterstützung die Trump-Administration kurzerhand im Frühjahr 2020 einstellte.

19 Häde, in: Pechstein/Nowak/Häde, EUV/GRC/AEUV, Art. 311 AEUV, Rn. 18.

$20 \mathrm{Zu}$ denken ist hier insbesondere an Art. 310 Abs. 1 UAbs. 3, 310 Abs. 4 oder 311 Abs. 2 AEUV.

21 So z.B. Waldhoff, in: Calliess/Ruffert, EUV/AEUV, Art. 311 AEUV, Rn. 17 oder Magiera, in: Grabitz/Hilf/Nettesheim, Recht der EU, Art. 311 AEUV, Rn. 23.

22 So Häde, in: Pechstein/Nowak/Häde, EUV/GRC/AEUV, Art. 311 AEUV, Rn. 16 ff. 
denfalls aber eine Finanzierung des allgemeinen Haushalts durch die Aufnahme von Fremdkapital verboten ist. ${ }^{23}$ Vor diesem Hintergrund würde - aus dieser Sicht - die Kapitalaufnahme der NGEU durch Eigenmittel rechtlichen Bedenken begegnen.

Dazu ist indessen sogleich festzuhalten: Die für die NGEU aufgenommenen Finanzmittel sollen gerade nicht als Eigenmittel aufgenommen werden und gerade nicht zur Finanzierung des allgemeinen Haushalts der Union zur Verfügung stehen. Vielmehr soll es sich bei diesen Finanzmitteln - soweit sie nicht für Darlehen an die Mitgliedstaaten gem. Art. 2 Abs. 2 lit. b) Aufbauinstrument-VO vorgesehen sind um externe zweckgebundene Einnahmen handeln (Art. 3 Abs. 1 Aufbauinstrument-VO). Dies bedeutet, dass die Beiträge zur Finanzierung des Aufbauinstruments nicht als Eigenmittel im Unionshaushalt aktiviert und damit nicht zur freien Verfügbarkeit zugeteilt werden, sondern außerhalb des Haushalts ausschließlich zweckgebunden i.S.v. Art. 7 Abs. 2 lit. e Haushaltsordnung der Union ${ }^{24}$ für die in Art. 2 Abs. 2 lit. a) und c) Aufbauinstrument-VO genannten Programme und Zwecke geführt werden. ${ }^{25}$ Derartige externe zweckgebundenen Einnahmen stellen keine Eigenmittel dar, sondern gehören zur Gruppe der ,sonstigen Einnahmen“i.S.v. Art. 311 Abs. 2 AEUV. ${ }^{26}$

Soweit es sich um Finanzmittel zur Bereitstellung von Darlehen an die Mitgliedstaaten gem. Art. 2 Abs. 2 lit. b) Aufbauinstrument-VO handelt, handelt es sich nicht um externe zweckgebundene Einnahmen, sondern um andere sonstige Einnahmen, welche gem. Art. 4 Eigenmittelbeschluss ebenfalls nicht zur Finanzierung operativer Ausgaben freigegeben sind.

Sehr deutlich wird die Abgrenzung der für NGEU aufzunehmenden Finanzmittel von Eigenmitteln auch schon innerhalb der Systematik des neuen Eigenmittelbeschlusses: So werden in Art. 2 Eigenmittelbeschlusses - Eigenmittelkategorien und konkrete Methoden für ihre Berechnung - die Kategorien der Eigenmittel geregelt, u.A. auch die neue „Plastik-Abgabe“, siehe Art. 2 Abs. 1 lit. c) Eigenmittelbeschluss. Die Mittel für die Aufbauinstrumente werden jedoch erst - und gesondert - in Art. 5 des Eigenmittelbeschlusses aufgeführt.

Problematisch könnte gleichwohl sein, dass die Möglichkeit der Kapitalaufnahme an den Kapitalmärkten für externe zweckgebundene Einnahmen auf den Kompetenztitel Art. 311 Abs. 3 AEUV zum System der Eigenmittel gestützt wird, obwohl es sich eben nicht um Eigenmittel handelt. Überdies: Wie der Vergleich zur Finanzierung des EFSM verdeutlicht, steht die Verortung der Kapitalaufnahme in Art. 311 Abs. 3 AEUV auch nicht in Einklang mit der damalig gewählten Struktur, findet somit auch kein historisches Vorbild. Gemäß des auf Art. 122 Abs. 2 AEUV ge-

23 Nachweise dazu bei Magiera, in: Grabitz/Hilf/Nettesheim, Recht der EU, Art. 311 AEUV, Rn. 23; auch bereits Seidel, RIW 1977, 665 (666); Waldhoff, in: Calliess/Ruffert, EUV/ AEUV, Art. 311 AEUV, Rn. 17.

24 Verordnung (EU, Euratom) 2018/1046 des Europäischen Parlaments und des Rates vom 18. Juli 2018 über die Haushaltsordnung für den Gesamthaushaltsplan der Union, AB1. L 193 v. 30.7.2018, 1.

25 Anders der Bundesrechnungshof in einem auf $\S 99$ BHO gestützten Bericht zu NGEU v. 11.3.2021 S. 20, BT- Drs. 19/27695, welcher unzutreffenderweise die Rechtmäßigkeit der Schuldenfinanzierung insbesondere am Haushaltsausgleichserfordernis aus Art. 310 Abs. 1 AEUV misst.

26 Rossi, in: Vedder/Heintschel von Heinegg, Europäisches Unionsrecht, Art. 311 AEUV, Rn. 8. 
stützten Art. 2 iVm. Art. 3 EFSM-VO wurde seinerzeit die Kommission mit Ratsbeschluss ermächtigt, Kapital am Kapitalmarkt aufzunehmen. Für die Finanzierung des EFSM wurde folglich nicht auf eine Regelung im Rahmen des Systems der Eigenmittel nach Art. 311 Abs. 3 AEUV zurückgegriffen, sondern die Kapitalaufnahme ausschließlich auf die Durchführungskompetenz aus Art. 122 AEUV gestützt. Stellt der auf Art. 311 Abs. 3 AEUV gestützte Eigenmittelbeschluss deshalb die falsche Kompetenzgrundlage für die Kapitalaufnahme dar und hätte diese richtigerweise in der auf Art. 122 AEUV gestützten Aufbauinstrument-VO geregelt werden müssen?

\section{b) Kapitalrückführung, Art. 5 Abs. 2 Eigenmittelbeschluss}

Unter Verweis auf die historischen Vorbilder wie EFSM oder Zahlungsbilanzanleihen mag das abweichende Vorgehen im Rahmen der Kapitalaufnahme der NGEU inkohärent erscheinen. Inkohärenz ist indessen eine Frage der Referenzargumente. Vorliegend gilt es zu berücksichtigen, dass NGEU im Vergleich zum EFSM oder den Zahlungsbilanzanleihen eine weitere Ebene beschreitet: Anders als in den historischen Vergleichsfällen ist NGEU von Anfang an und ganz bewusst so konzipiert, dass es auf Ebene der Kapitalrückführung zu einem Finanzierungsdefizit kommen wird, indem die NGEU-Programme verlorene Zuschüsse in Form nicht rückzahlbaren Unterstützungen vorsehen. Damit ist unmittelbar klar, dass für NGEU auf Ebene der Kapitalrückführung ein zusätzlicher Kapitalbedarf in Höhe des konkreten Finanzierungsdefizits entstehen wird. Dieser soll durch den allgemeinen Haushalt gedeckt werden, Art. 5 Abs. 2 UAbs. 1 des Eigenmittelbeschlusses:

„,Die Rückzahlung des Kapitalbetrags der Mittel, die für die in Unterabsatz 1 Buchstabe $b$ des vorliegenden Artikels genannten Ausgaben zu verwenden sind, und die dafür fälligen Zinsen gehen zulasten des Haushaltsplans der Union. "

Dieser zusätzliche Finanzierungsbedarf - in toto bis zu 390 Mrd. $€$ nebst Zinsen bis 2058, Art. 5 Abs. 2 UAbs. 2 Eigenmittelbeschluss - darf jedoch den allgemeinen Haushalt der Union mit Blick auf haushaltsrechtliche Grundsätze wie den des Haushaltsausgleichs aus Art. 310 Abs. 1 UAbs. 3 AEUV und den der Haushaltsdisziplin aus Abs. 4 nicht unverhältnismäßig überstrapazieren und einschränken. Deshalb sind auf Ebene der Kapitalrückführung zusätzliche Sicherungen vorzusehen, welche die Gewährleistung dieser Grundsätze garantieren.

Dazu soll ein besonderes Rückzahlungsmanagement, Art. 5 Abs. 2 UAbs. 2 Eigenmittelbeschluss, eingerichtet werden, welches eine gleichmäßige und nicht überbordende Inanspruchnahme der Haushaltsmittel gewährleisten soll. Zur absoluten Sicherung der jederzeitigen Bedienung des Kapitaldienstes erfolgt die Gewährleistung ausreichender - zusätzlicher - Finanzmittel für den Haushalt unter Anderem mittels einer temporären Anhebung der Eigenmittelobergrenzen (Art. 6 Eigenmittelbeschluss) und der kurzfristigen Einstands- und Nachschusspflicht der Mitgliedstaaten bei Liquiditätslücken (Art. 9 Abs. 4 Eigenmittelbeschluss). Zwar führt die temporäre Anhebung der Eigenmittelobergrenze in Summe zu einer Übersicherung der für NGEU aufge- 
nommenen Finanzmittel. ${ }^{27}$ Dies bezweckt mittels bester Bonität bestmögliche Kreditkonditionen am Markt zu erhalten. Dazu ist die deutliche Übersicherung erforderlich, weil nicht jeder Mitgliedstaat über ein sehr gutes Bonitätsrating verfügt. Insgesamt resultiert aus dieser Übersicherung aber kein strukturelles Problem der überbordenden Inanspruchnahme einzelner Mitgliedstaaten, weil gleichzeitig Art. 6 und Art. 9 Abs. 5 Eigenmittelbeschluss genau vorgeben, in welchem Umfang jedes Mitglied auf Ebene der Kapitalrückführung in Anspruch genommen werden darf. Die Übersicherung ist deshalb einzig aus der ex-ante Perspektive relevant, dem Markt glaubhaft zu versichern, dass die Schulden in jedem Fall zurückgezahlt werden. Strikt zu trennen ist davon die ex-post Frage, wie das durch die nicht rückzahlbaren Unterstützungen entstehende Finanzierungsdefizit zu refinanzieren ist. Wie unten (dazu III.) dargelegt wird, ergibt sich daraus jedenfalls keine strukturelle fiskalische Überforderung einzelner Mitglieder.

Dass all diese Modalitäten nur sinnvoll und rechtlich wirksam - es sei insbesondere an die Erhöhung der Eigenmittelobergrenze und die Nachschusspflicht gedacht - im System der Eigenmittel verankert werden können, ist offensichtlich: Es handelt sich gerade um genuines Haushaltsrecht, wenn die Eigenmittelobergrenze angepasst oder geändert wird. Darüber hinaus würden die in Art. 311 Abs. 3 AEUV zugesicherten Partizipationsmöglichkeiten der Mitgliedstaaten und insbesondere der nationalen Parlamente beeinträchtigt werden, wenn derartige Änderungen im Haushaltsregime und insbesondere weitreichende Verpflichtungen der Mitgliedstaaten lediglich in der Ermächtigung des Basisrechtsaktes - also gestützt auf Art. 122 AEUV - und ohne deren Beteiligung geregelt würden. Daher kann letztlich kein berechtigter Zweifel bestehen, dass Art. 311 Abs. 3 AEUV jedenfalls die zutreffende Kompetenzgrundlage für die Regelungen der Kapitalrückführung darstellt.

Dass man auch die Kapitalaufnahme im Eigenmittelbeschluss abweichend von der historischen Praxis auf Art. 311 Abs. 3 AEUV gestützt hat, erscheint aufgrund des strengen Konnexes zwischen Kapitalaufnahme und -rückführung, aus Gründen der Klarheit sowie unter Berücksichtigung dessen, dass Art. 311 Abs. 3 AEUV das aus mitgliedstaatlicher Sich souveränitätsschonendere Verfahren vorsieht, weil es eine mitgliedstaatliche Ratifikation voraussetzt, durchaus sachdienlich und deswegen im Ergebnis richtig.

Entsprechend argumentiert auch der Rat, siehe dazu den 16. Erwägungsgrund im neuen Eigenmittelbeschluss:

„Die Ermächtigung der Kommission, im Namen der Union Mittel an den Kapitalmärkten zu dem alleinigen und ausschließlichen Zweck aufzunehmen, die Maßnahmen zur Bewältigung der Auswirkungen der COVID-19-Krise zu finanzieren, steht in engem Zusammenhang mit der in diesem Beschluss vorgesehenen Anhebung der Eigenmittelobergrenze und letztlich mit dem Funktionieren des Eigenmittelsystems der Union. Folglich sollte diese Ermächtigung in den vorliegenden Beschluss aufgenommen werden."

$27 \mathrm{Zu}$ drastisch der Bundesrechnungshof, Fn. 25, S. 29: „Eine Anhebung der Eigenmittelobergrenze, ausgerichtet an dem jährlich maximal zulässigen Rückzahlungsbetrag, führt damit zu einem strukturell überhöhten Garantievolumen.“. 


\section{c) Kapitalverwendung, Art. 2 Aufbauinstrument-VO}

Dass die Ebene der Kapitalverwendung nicht im Eigenmittelbeschluss, sondern über die Aufbauinstrument-VO auf Art. 122 AEUV gestützt geregelt wird, steht nicht nur in Einklang mit der bisherigen Praxis bei vergleichbaren Krisenbewältigungsmechanismen wie beispielsweise dem EFSM. Hier spiegelt sich auch, dass Art. 311 Abs. 3 AEUV keine Kompetenz zum konkreten Durchführen von Unterstützungsmaßnahmen darstellt. Damit kommt vorliegend ausschließlich Art. 122 AEUV als Rechtsgrundlage in Betracht.

Auffällig ist allerdings, dass NGEU in der Aufbauinstrument-VO abweichend vom nur auf Art. 122 Abs. 2 AEUV gestützten EFSM auf beide Absätze von Art. 122 AEUV verweist. Die Bezugnahme auf Art. 122 Abs. 1 und Abs. 2 AEUV erweist sich auch keinesfalls als ein redaktionelles Versehen. Insbesondere die Bezugnahme auf Art. 122 Abs. 1 AEUV mag zunächst überraschen, dokumentiert bei genauerer Betrachtung aber den durch NGEU erfolgenden Entwicklungsschritt: Sie manifestiert die in den Verträgen angelegten Krisenbewältigungs- und Weiterentwicklungspotentiale. Art. 122 Abs. 1 AEUV hatte bisher nämlich kaum praktische Relevanz erlangt. ${ }^{28}$ Seine Aktivierung ist auch, so wird von vielen vertreten, von einer restriktiven Auslegung zu begleiten und deshalb umstritten. ${ }^{29}$ Dies begründet sich durch seine Ausnahmestellung im Spannungsverhältnis europäischer und mitgliedstaatlicher wirtschaftspolitischer Kompetenzen. Im Ergebnis besteht mit Art. 122 Abs. 1 AEUV aber eine Kompetenzgrundlage für die Union auf deren Grundlage sie zur Bekämpfung der Folgen der Corona-Pandemie mit der Vorgabe und Finanzierung diverser konjunktur- und strukturpolitischer Programme tätig werden darf, wie sogleich unter (aa) zu zeigen ist.

$\mathrm{Zu}$ betonen ist dabei auch folgender Umstand, den die Kritik nicht selten außer Betracht lässt: Die eigentliche Umsetzung der in NGEU vorgesehenen Programme erfolgt gar nicht durch die Union selbst, sondern ist auf die Ebene der Mitgliedstaaten delegiert. Die Mitgliedstaaten entwerfen dazu Aufbau- und Resilienzpläne, nach denen sie im Einklang mit den im Rahmen des Europäischen Semesters ermittelten einschlägigen länderspezifischen Herausforderungen und Prioritäten mit der Kommission abgestimmte Investitionsprogramme aufsetzen. Die Union stellt zur Verwirklichung dieser Programme die notwendigen Finanzmittel durch die für NGEU geschaffene eingangs erwähnte ${ }^{30}$ Aufbau- und Resilienzfazilität bereit. Damit entfaltet NGEU nicht nur eine konjunktur- und strukturpolitische Dimension, sondern bewegt sich auch im Spannungsfeld finanziellen Beistands seitens der Union zugunsten der Mitgliedstaaten, so dass die Umsetzung von NGEU ebenfalls an den Voraussetzungen

28 Vgl. lediglich diverse Richtlinien zur Mineralölversorgung wie 68/414/EWG, 73/238/ EWG, 2006/67/EG und 2009/119/EG.

29 S. etwa Rodi, in: Vedder/Heintschel von Heinegg, Europäisches Unionsrecht, Art. 122 AEUV, Rn. 3; Bandilla, in: Grabitz/Hilf/Nettesheim, Recht der EU, Art. 122 AEUV, Rn. 11; im Ergebnis auch so Häde, in: Calliess/Ruffert, EUV/AEUV, Art. 122 AEUV, Rn.2f.; besonders deutlich Kempen, in: Streinz, EUV/AEUV, Art. 122 AEUV, Rn. 3; a.A. Smulder/Keppenne, in: von der Groeben/Schwarze/Hatje, Europäisches Unionsrecht, Art. 122 AEUV, Rn. 6 f.

30 S. oben Fn, 10. 
von Art. 122 Abs. 2 AEUV zu messen ist. NGEU erfüllt aber auch diese spezielleren Voraussetzungen, dazu (bb). Doch der Reihe nach.

Zunächst empfiehlt es sich, sich einen kurzen Überblick über die Umsetzung und Durchführung der für NGEU in Art. 2 Abs. 2 Aufbauinstrument-VO vorgesehenen Programme $\mathrm{zu}$ verschaffen. Beides wird in der Aufbauinstrument-Durchführungs$\mathrm{VO}^{31}$ geregelt. Darin wird eine Aufbau- und Resilienzfazilität eingerichtet, welche die für NGEU vorgesehenen Unterstützungen bereitstellen wird, Art. 1. Als Ziel formuliert die Verordnung, ,den Mitgliedstaaten finanzielle Unterstützung für die Verwirklichung der in ihren Aufbau- und Resilienzplänen festgelegten Etappenziele und Zielwerte ihrer Reformen und Investitionen zur Verfügung zu stellen“, Art. 4 Abs. 2. Dazu erfolgt gemäß Art. 12 die Zuweisung der nicht rückzahlbaren Unterstützungen und gemäß Art. 14 Aufbauinstrument-Durchführungs-VO die Gewährung der Darlehen zugunsten der Mitgliedstaaten. Darüber hinaus regelt die Aufbauinstrument-Durchführungs-VO die Voraussetzungen für die Gewährung der jeweiligen Unterstützungen, Art. 17 f., das Beurteilungs- und Annahmeverfahren, Art. 19 f., Maßnahmen zur Verknüpfung der Fazilität mit einer ordnungsgemäßen wirtschaftspolitischen Steuerung sowie Kontrollmechanismen zur Überprüfung der Kohärenz mitgliedstaatlicher Maßnahmen mit den eingereichten Aufbau- und Resilienzplänen, Art. 10 Aufbauinstrument-Durchführungs-VO.

\section{aa) Struktur- und Konjunkturprogramme, Art. 122 Abs. 1 AEUV}

Kernelement von NGEU ist, dass die Union konjunktur- und strukturpolitisch tätig wird, indem sie den Mitgliedstaaten notwendige und abgestimmte Investitionsprogramme durch Unionsmittel finanziert. ${ }^{32}$ Dazu sieht NGEU 390 Mrd. $€$ nicht rückzahlbarer sowie 360 Mrd. € rückzahlbarer Unterstützungen für diverse Programme vor, die vornehmlich den Zweck der Stärkung der sozialen und wirtschaftlichen Resilienz verfolgen. Indem es sich hierbei um das Auflegen von konkreten Programmen unter Vorgabe konkreter Zielparameter im Rahmen der Aufbau- und Resilienzpläne handelt, ist ein alleiniger Rückgriff auf die tradierte Kriseninterventionskompetenz aus Art. 122 Abs. 2 AEUV als Kompetenznorm verbaut. Vielmehr bedarf es zusätzlich einer Norm, welche der Union gestattet, insbesondere in den in Art.2 Abs. 2 lit. a) Aufbauinstrument-VO genannten Bereichen wie Struktur- und Kohäsionspolitik, Reformen und Investitionen, Forschung und Innovation, Klimapolitik oder der Entwicklung ländlicher Gebiete tätig zu werden. Gesucht wird eine Kompetenznorm, welche die Union zum krisenbedingten konjunktur- und strukturpolitischen Tätigwerden ermächtigt, so dass diese neben den mitgliedstaatlichen Anstrengungen mit einem wirtschaftspolitischen Impuls die Wirtschaft in der Union stimulieren kann. An diesen Anforderungen muss sich die gewählte Rechtsgrundlage messen lassen. ${ }^{33}$

Hier hat der europäische Gesetzgeber Art. 122 Abs. 1 AEUV ausgemacht. Dieser ermächtigt den Rat, auf Vorschlag der Kommission unbeschadet der sonstigen in den

31 Verordnung (EU) 2021/241 des Europäischen Parlaments und des Rates vom 12. Februar 2021 zur Einrichtung der Aufbau- und Resilienzfazilität.

32 S. die ausführliche Analyse der NGEU-Maßnahmen in einem Gutachten des Juristischen Dienstes des Rates, Rats-Dok. Nr. 9062/20 v. 24.6.2020, Rn. 139 ff.

33 Ebd., Rn. 119. 
Verträgen vorgesehenen Verfahren im Geiste der Solidarität zwischen den Mitgliedstaaten über die der Wirtschaftslage angemessenen Maßnahmen zu beschließen, insbesondere falls gravierende Schwierigkeiten in der Versorgung mit bestimmten Waren, vor allem im Energiebereich, auftreten. Es ist zu fragen, ob im vorliegenden Kontext die Tatbestandsvoraussetzungen erfüllt sind und die Subsidiaritäts- wie die Verhältnismäßigkeitsanforderungen eingehalten werden.

Die tatbestandlichen Voraussetzungen für mögliche der Wirtschaftslage angemessenen Maßnahmen werden in Art. 122 Abs. 1 AEUV allerdings nicht gerade klar formuliert: Als einzig greifbare Voraussetzung stellt sich die beispielhafte Bezugnahme auf „gravierende Schwierigkeiten in der Versorgung mit bestimmten Waren, vor allem im Energiebereich" dar. ${ }^{34}$ Das Destillat dieser Formulierung sei, so wird vertreten, dass ein Eingreifen auf Grundlage von Art. 122 Abs. 1 AEUV jedenfalls nur im Falle wirtschaftlicher Schwierigkeiten in Betracht kommen könne, jedoch darüber hinaus tatbestandlich der Anwendungsbereich nicht weiter eingegrenzt sei. ${ }^{35}$ Der Juristische Dienst des Rates bringt es in seinem Gutachten zur Rechtmäßigkeit von NGEU auf die Formel, dass dem Tätigwerden eine „Notsituation oder eine(r) außergewöhnliche Situation“" zugrunde liegen und es sich „um Maßnahmen wirtschaftlicher Art handeln“ muss. ${ }^{36}$ Diese Sicht verdient grundsätzlich Zustimmung. Insbesondere die Formulierung „im Geiste der Solidarität zwischen den Mitgliedstaaten“ und die Verbindung zu Abs. 2 bestätigen dies. Solidarität wird typischerweise gerade in Krisenzeiten notwendig bzw. eingefordert. Zu betonen ist zugleich, dass anders als in Abs. 2 die Anforderungen an eine durch Abs. 1 adressierte Krise aber nicht konkretisiert werden. Es ist eben lediglich von etwaigen „Schwierigkeiten“ die Rede. Parallel zu Abs. 2 ist jedoch auch Abs. 1 nur dann aktivierbar, wenn solche Schwierigkeiten vorliegen. Beide Absätze handeln mit unterschiedlichen Nuancen von Krisenmechanismen. Aus der Bezugnahme auf diese folgt deshalb auch, dass es sich bei auf Art. 122 Abs. 1 AEUV gestützten Maßnahmen ausschließlich um zeitlich befristete und damit nicht dauerhafte Maßnahmen handeln darf. Dass es sich bei der Bekämpfung der wirtschaftlichen Folgen der Pandemie um den Umgang mit Schwierigkeiten handelt, ist ob des historischen Ausmaßes der Pandemie unstreitig. Die Befristung des Programms wird dadurch gewährleistet, dass die nicht rückzahlbaren Mittel gemäß Art. $12 \mathrm{zu} 70 \%$ bis zum 31. Dezember 2022 und die restlichen 30\% bis zum 31. Dezember 2023 sowie die Darlehen gemäß Art. 14 Aufbauinstrument-Durchführungs-VO bis zum 31. Dezember 2023 und ausnahmsweise bis zum 31. August 2023 zur Verfügung gestellt worden sein müssen.

Art. 122 Abs. 1 AEUV erlaubt es dem Rat also, „angemessene Maßnahmen“ beschließen zu können. Dem Rat wird dazu ein sehr weites Ermessen hinsichtlich des „Ob“ und des „Wie“ bezüglich möglicher Maßnahmen eingeräumt. ${ }^{37}$ Im Schrifttum

34 Ebenfalls Smulder/Keppenne, in: von der Groeben/Schwarze/Hatje, Europäisches Unionsrecht, Art. 122 AEUV, Rn. 6.

35 Ebenso Herrmann/Dausinger, in: Frankfurter Kommentar, EUV/GRC/AEUV, Art. 122 AEUV, Rn. 9.

36 Gutachten des Juristischen Dienstes des Rates, oben Fn. 32, Rn. 121.

37 Herrmann/Dausinger, in: Frankfurter Kommentar, EUV/GRC/AEUV, Art. 122 AEUV, Rn. 10; dies wird auch in historischer Sicht untermauert, wie der Vergleich zu den Vorgängernormen zeigt, dazu Häde, in: Calliess/Ruffer, EUV/AEUV, Art. 122 AEUV, Rn. 1. 
wird indessen auch vertreten, dass die Reichweite der Aktivierung von Art. 122 Abs. 1 AEUV gleich aus mehreren Gründen einzugrenzen sein soll: Erstens dürfe die ,grundsätzliche Zuständigkeit der Mitgliedstaaten für die allgemeine Wirtschaftspolitik nicht in Frage gestellt werden“. ${ }^{38}$ Zweitens ergebe sich aus dem systematischen Zusammenhang zu insbesondere Art. 121 und Art. 126 AEUV, dass die dort vorgesehenen Instrumente nicht mittels der leichteren Anforderungen des Art. 122 Abs. 1 AEUV unterwandert werden dürften. ${ }^{39}$ Drittens und letztens impliziere die Formulierung ,unbeschadet der sonstigen in den Verträgen vorgesehenen Verfahren“ eine Subsidiarität von Art. 122 Abs. 1 AEUV gegenüber spezifischeren Politikbereichen. ${ }^{40}$

In Hinblick auf die rechtliche Beurteilung von NGEU ergeben sich daraus indessen keine durchgreifenden Einwände:

Zunächst konkurriert der hier in Rede stehende Teil von NGEU schon gar nicht mit den in Art. 121 und Art. 126 AEUV vorgesehenen Instrumenten. Die Programme sehen keine Art. 121 AEUV entgegenstehenden Koordinierungsanweisungen zulasten mitgliedstaatlicher wirtschaftspolitischer Souveränität vor, sondern setzen gerade voraus, dass die unter NGEU geplanten Investitionsprogramme in kohärenter Weise zu den Vorgaben des Europäischen Semesters durchgeführt werden, Art. 17 Abs. 3 Aufbauinstrument-Durchführungs-VO. Auch wirken sie nicht auf die Haushaltsgrundsätze aus Art. 126 AEUV ein, sondern bedingen ein Einhalten dieser, Art. 10 Aufbauinstrument-Durchführungs-VO. Beide Normen werden durch NGEU also nicht unterminiert, sondern vielmehr gestärkt. Eine für Art. 122 Abs. 1 AEUV angenommene Subsidiarität wird ebenfalls nicht berührt: Es ist schon keine konkurrierende Norm ersichtlich, welche mit NGEU unterlaufen werden könnte. Dass man sich die Frage überhaupt stellt, ob Art. 122 Abs. 1 AEUV eine taugliche Kompetenzgrundlage abgibt, resultiert gerade aus der Erkenntnis, dass eine andere, ähnliche Kompetenzen vorsehende Norm nicht in Reichweite ist. Außerdem lässt sich bereits fragen, ob Art. 122 Abs. 1 AEUV die ihm gelegentlich zugeschriebene Subsidiarität überhaupt enthält. Mit Blick auf die Formulierung ,im Geiste der Solidarität“ lässt sich nämlich gut vertreten, dass eine derartige Subsidiaritätsannahme den Stellenwert der Solidarität zwischen den Mitgliedstaaten verkennt. Aus dem Wortlaut lässt sich jedenfalls keine strenge Subsidiarität ableiten. ${ }^{41}$ Dies wird durch die Rechtsprechung zu Art. 18 AEUV gestützt, wo die Formulierung ,unbeschadet besonderer Bestimmungen der Verträge" an Art. 122 Abs. 1 AEUV erinnert: Dort zieht der EuGH Art. 18 Abs. 1 AEUV und die einschlägigen besonderen Bestimmungen ohne weiteres

38 Häde, in: Calliess/Ruffer, EUV/AEUV, Art. 122 AEUV, Rn. 2.

39 Kempen, in: Streinz, EUV/AEUV, Art. 122 AEUV, Rn. 3; ebenfalls Häde, in: Calliess/ Ruffert, EUV/AEUV, Art. 122 AEUV, Rn. 5: „Abs. 1 tritt hinter speziellere Befugnisse zurück, die Einwirkungen auf die mitgliedstaatliche Wirtschaftspolitik vorsehen.“; Bandilla, in: Das Recht der Europäischen Union, Art. 122 AEUV, Rn. 11.

40 Bandilla, in: Grabitz/Hilf/Nettesheim, Recht der EU, Art. 122 AEUV, Rn. 11 unter Verweis auf EuGH, Rs. 31/74, Galli, Slg. 1975, 47, 63; etwas zurückhaltender, im Ergebnis aber auch so Rodi, in: Vedder/Heitschel von Heinegg, Europäisches Unionsrecht, Art. 122 AEUV, Rn. 3.

41 Wohl auch in diese Richtung Smulder/Keppenne, in: von der Groeben/Schwarze/Hatje, Europäisches Unionsrecht, Art. 122 AEUV, Rn. 9. 
nebeneinander heran. ${ }^{42}$ Eine strenge Subsidiaritätsannahme bei Verwendung der besagten Formulierung findet in der Rechtsprechung des Gerichtshofs mithin keine Stütze. Zu Art. 122 Abs. 1 AEUV besteht jedenfalls gar keine belastbare Rechtsprechung, welche eine Subsidiarität zwingend vorschreibt. Das einzige aus der Rechtsprechung des EuGH destillierte Argument für eine Subsidiarität von Art. 122 Abs. 1 AEUV bezieht sich auf ein Urteil, welches noch zur Vorgängernorm Art. 103 EWG-Vertrag erging. Dabei ist aber sehr fraglich, ob es dort um Nachrangigkeit oder Subsidiarität ging. Mitgliedstaaten wurde die Berufung auf die auf Art. 103 EWG-Vertrag gestützte gemeinschaftliche Konjunkturpolitik versagt, weil der zugrundeliegende Sachbereich - Agrarmärkte - bereits vergemeinschaftet war. Womöglich war dies aber nur die Feststellung, dass die allgemeinere Norm (Art. 103 EWGVertrag) die speziellere (Agrarmarktpolitik) nicht verdrängt. Auch vorliegend würden die krisenbedingten Maßnahmen neben etwaigen bereits bestehenden unionalen Maßnahmen stehen, sie jedenfalls nicht ersetzen. Die Kohärenz mit der Politik der Union in anderen Bereichen wird durch NGEU deshalb ausdrücklich in den Blick genommen, Art. 1 Abs. 3 Aufbauinstrument-VO.

Auch aus den allgemeinen Subsidiaritätsanforderungen unionalen Handels aus Art. 5 Abs. 3 EUV ergeben sich für das Verhältnis von NGEU zur grundsätzlichen Zuständigkeit der Mitgliedstaaten für die allgemeine Wirtschaftspolitik keine Einwände: Ausweislich der Begründung für den Vorschlag zur Aufbauinstrument-VO sollen die Programme die mitgliedstaatlichen Bemühungen ergänzen, keinesfalls ersetzen. ${ }^{43} \mathrm{Al}-$ les andere wäre zirkulär: NGEU wird doch gerade deshalb aufgesetzt, weil mitgliedstaatliche Anstrengungen nicht mehr auszureichen drohen, um ernstlichen und dauerhaften ökonomischen Schaden in den nationalen Volkswirtschaften und damit im Binnenmarkt zu verhindern. NGEU zielt von vornherein auf ein „Nebeneinander“ statt eines „Stattdessen“ ab und wird nur deshalb aufgesetzt, weil die mitgliedstaatlichen Mittel eben nicht ausreichen. Entsprechend spielen die Mitgliedstaaten bei der konkreten Umsetzung von NGEU eine zentrale Rolle: Sie entwickeln in Zusammenarbeit mit der Kommission die konkreten Aufbau- und Resilienzpläne, auf Grundlage derer die konkreten Investitionen getätigt werden, Art. 18 Aufbauinstrumet-DurchführungsVO.

Nach all dem ist zusammenzufassend festzustellen, dass NGEU auf Art. 122 AEUV Abs. 1 gestützt werden kann. Der Union ist in den Verträgen die Kompetenzgrundlage an die Hand gegeben worden, in Zeiten der Krise im Geiste der Solidarität konjunktur- und strukturpolitisch tätig zu werden - so denn die politischen Mehrheiten gegeben sind.

Auf der Ebene der Ausübung dieser Zuständigkeit bestehen ebenfalls keine Bedenken: Ein zusätzliches Tätigwerden zum Wohle der Unionsbürgerinnen und Unionsbürger und - konkreter - im Sinne eines raschen Setzens eines signifikanten Stimulus ist geboten. Aufgrund versiegender mitgliedstaatlicher Potentiale und aufgrund der anhaltenden akuten Bedrohungslage durch die Pandemie sind solche im Geiste der

42 Ausführlich dazu Epiney, in: Calliess/Ruffert, EUV/AEUV, Art. 18 AEUV, Rn. 4 f.

43 S. die Begründung zur Rechtsgrundlage, Subsidiarität und Verhältnismäßigkeit im Vorschlag über eine Verordnung des Rats zur Schaffung eines Aufbauinstruments der Europäischen Union zur Unterstützung der Erholung nach der COVID-19-Pandemie, COM(2020) 441 final (Aufbauinstrument-VO) v. 28.5.2020, S. 3. 
Solidarität auf Unionsebene zu aktivieren. Dies erfolgt mit NGEU. Gleichzeitig gehen von NGEU keine substanziellen Kompetenzverschiebungen zulasten der Mitgliedstaaten aus. NGEU ist zeitlich und inhaltlich klar begrenzt. ${ }^{44}$ NGEU ist damit auch verhältnismäßig:

„Die erforderliche Höhe der Finanzierungen aus dem Aufbauinstrument der Europäischen Union lässt sich durch die derzeit in der Union bestehenden beispiellosen Umstände begründen. Das Aufbauinstrument ist im Hinblick auf seine Dauer und seine Verwendung klar begrenzt. " 45

Dem ist nichts hinzuzufügen.

\section{bb) Finanzielle Unterstützungen, Art. 122 Abs. 2 AEUV}

Indem die Aufbau- und Resilienzfazilität die Mitgliedstaaten in Abstimmung mit der Kommission mit der Umsetzung der konkreten Investitionsprogramme betraut und ihnen dazu nicht rückzahlbare und rückzahlbare Unterstützungen zuteilt, sind die Maßnahmen der Mittelverwendung von NGEU auch an den Maßstäben von Art. 122 Abs. 2 AEUV zu messen.

Gemäß Art. 122 Abs. 2 AEUV darf der Rat den Mitgliedstaaten finanzielle Beistand in besonderen Krisensituationen und unter bestimmten Bedingungen seitens der Union gewähren. Damit ist Abs. 2 ausdrücklich auf finanziellen Beistand zugunsten der Mitgliedstaaten zugeschnitten und mithin spezieller als Abs. $1 .^{46}$

Vor diesem Hintergrund sind für jegliche Formen finanziellen Beistands wie rückzahlbare oder nicht rückzahlbare Unterstützungen zugunsten der Mitgliedstaaten die engeren Voraussetzungen des Abs. 2 einzuhalten. Eine Umgehung durch den Rückgriff auf nur Abs. 1 wäre in solchen Konstellationen normwidrig. Damit darf finanzieller Beistand nur dann gewährt werden, wenn der Mitgliedstaat aufgrund eines Krisenmomentums von Schwierigkeiten betroffen oder von gravierenden Schwierigkeiten ernstlich bedroht wird.

Ein solches Krisenmomentum i.S.v. Art. 122 Abs. 2 AEUV kann eine Naturkatastrophe oder ein außergewöhnliches Ereignis sein, das sich der mitgliedstaatlichen Kontrolle entzieht. Dass es sich bei der derzeit wütenden Pandemie um ein solches Krisenmomentum handelt, ist ernsthaft nicht $\mathrm{zu}$ bestreiten und bedarf keiner vertieften Ausführungen. Ob die Pandemie als eine Naturkatastrophe oder als ein außergewöhnliches Ereignis einzuordnen ist, welches sich der mitgliedstaatlichen Kontrolle entzieht, kann dahinstehen: selbst wenn man die Pandemie nicht als eine Naturkata-

44 Soweit dies bestritten wird, gründet sich dies regelmäßig auf haltlose Mutmaßungen und spekulative Annahmen unter Außerachtlassung der Singularität der Pandemie, so etwa jüngst der Bundesrechnungshof (oben Fn. 25): „Aller Erfahrung nach [sic!] verstetigen sich auf EU-Ebene in Krisenzeiten einmal eingeführte Instrumente eher, als dass ihre Anwendung auf den Krisenfall beschränkt bleibt. Auch die angestrebte Tilgung der EU-Anleihen durch neue EU-Eigenmittel legt nahe, dass mit dem Wiederaufbaufonds dauerhafte fiskalische Integrationsschritte verbunden sein könnten.".

$45 \operatorname{COM}(2020) 441$ final v. 28.5.2020 (oben Fn. 2), S. 3, dort auch bereits die Begründung zur Rechtsgrundlage, Subsidiarität und Verhältnismäßigkeit von NGEU.

46 Klarstellend EuGH, Rs. Pringle, C-370/12 ECLI:EU:C:2012:756, Rn. 116. 
strophe einordnen würde, so wäre sie jedenfalls ein qualifiziertes außergewöhnliches Ereignis im Sinne von Art. 122 Abs. 2 AEUV.

Dass wegen dieses Ereignisses die Mitgliedstaaten von Schwierigkeiten betroffen sind, legen bereits die in historischem Umfang aufgelegten mitgliedstaatlichen Rettungsmaßnahmen nahe. Man kann sich freilich fragen, ob diese Form von Betroffenheit hinreicht oder finanzieller Beistand nicht doch erst dann gewährt werden kann, wenn ein Mitgliedstaat sich selbst zu helfen außer Stande ist. ${ }^{47}$ Letzteres wird und wurde insbesondere in der Diskussion um die Rechtmäßigkeit des EFSM zu Art. 122 Abs. 2 AEUV mit Verweis auf Art. 125 AEUV und die dort begründete Stabilitätskonzeption der WWU vertreten. ${ }^{48} \mathrm{Zu}$ fragen ist, ob Art. 122 Abs. 2 AEUV eine derartige Subsidiarität zwingend vorsieht und deshalb Beistand verwehrt ist, wenn der „Worst case“ noch nicht eingetreten ist.

Dass auf Art. 122 Abs. 2 AEUV gestützter Beistand lediglich als letztes Mittel gewährt werden soll, steht indessen genau besehen schon im Widerspruch zur Norm: Erstens ergibt sich ein Ultima ratio-Charakter schon aus dem Wortlaut nicht, weil finanzieller Beistand auch bereits dann gewährt werden darf, wenn - insoweit qualifiziert - gravierende Schwierigkeiten ernstlich drohen. Zweitens steht eine solche Vorstellung - solidarische Hilfe erst als allerletzter Ausweg - mit dem Sinn und Zweck der Regelung, sich im Zeitpunkt eines Krisenmomentums mit dem betroffenen Mitglied solidarisch zu zeigen, in Widerspruch zu dem in Art. 122 Abs. 2 AEUV zugrunde gelegten Solidaritätsgedanken. Drittens verkennt die Forderung, finanziellen Beistand erst als ultima ratio zu zulassen, das Verhältnis von Art. 122 zu Art. 125 AEUV. Art. 122 Abs. 2 AEUV stellt keinesfalls eine Art Ausnahme zu Art. 125 AEUV dar, weshalb ersterer restriktiv auszulegen wäre. Vielmehr stehen beide Normen hierarchisch und konzeptionell nebeneinander, Art. 122 AEUV systematisch gar vor Art. 125 AUEV, was ebenfalls gegen ein Regel-Ausnahmeverhältnis spricht. ${ }^{49}$ Letztlich handeln beide Artikel von in Art. 3 EUV genannten Zielen der Union - von Stabilität und Solidarität. Hier einen Vorrang des einen Ziels ausmachen zu wollen, erscheint nicht sachgerecht.

Gerade vor dem Hintergrund der Erfahrungen der Dynamiken der Schuldenkrise zu Beginn der 2010er Jahre täte Europa in dieser Krise außerdem gut daran zu verhindern, dass staatliche Krisenbewältigungsmaßnahmen die jeweiligen Finanzierungspo-

47 In diese Richtung unter Bezugnahme auf die insbesondere in Art. 125 AEUV zum Ausdruck kommende haushaltsökonomische Eigenverantwortung der Mitgliedstaaten jüngst der Bundesrechnungshof, der - unzutreffenderweise - die Notwendigkeit unionalen Handelns im Rahmen von NGEU ablehnt, oben Fn. 25, S. 19.

48 So Häde, EuZW 2009, 399 (404); auch Tietje, ifo Schnelldienst 4/2021, 16 (19): „Es bleibt insofern unabdingbar zunächst immer bei der Selbstverantwortung der Staaten der Eurogruppe für ihre Wirtschaftspolitik. Finanzielle Solidariätsmassnahmen müssen die Ultima Ratio im Euroraum bleiben.“.

49 In diesem Sinne wohl EuGH, Rs. Pringle, C-370/12 ECLI:EU:C:2012:756, Rn. 131: „Würde Art. 125 AEUV jede finanzielle Unterstützung der Union oder der Mitgliedstaaten für einen anderen Mitgliedstaat verbieten, hätte in Art. 122 AEUV klargestellt werden müssen, dass er eine Ausnahme von Art. 125 AEUV darstellt"; ebenfalls gegen ein Ausnahmeverhältnis Herrmann/Dausinger, in: Frankfurter Kommentar, EUV/GRC/AEUV, Art. 125 AEUV, Rn. 17; oder Calliess, Das europäische Solidaritätsprinzip und die Krise des Euro, Vortrag an der Humboldt-Universität zu Berlin am 18.1.2011, FCE 1/11, 42 ff. 
tentiale überstrapazieren und sicherzustellen, dass die Mitgliedstaaten nicht wieder durch dramatisch steigende Refinanzierungskosten und Zinsspreads in eine finanzielle Isolation getrieben werden, wie es zu Beginn der europäischen Schuldenkrise zu beobachten war. Gewiss darf dies nicht $\mathrm{zu}$ einer Übergehung der in Art. 122 Abs. 2 AEUV vorgegebenen Voraussetzungen führen. Insgesamt kann aber wohl mit Blick auf den Anstieg der Gesamtverschuldung und der Arbeitslosenquoten in Europa sowie aufgrund des Andauerns der Pandemie nicht ernstlich bestritten werden, dass die Mitgliedstaaten von Schwierigkeiten betroffen oder ernstlich bedroht sind. Dass der Beistand der Union das letzte Mittel darstellen muss, ist wie dargelegt nicht erforderlich.

Zuletzt ist der Beistand „unter bestimmten Bedingungen“ zu gewähren. Was darunter zu verstehen ist, ist offen. Jedenfalls soll der Rat über einen weiten Spielraum verfügen, diese Bedingungen auszugestalten. Klar sollte jedoch sein, dass vor dem Hintergrund der Wechselwirkung mit Art. 125 AEUV die Bedingungen im Verhältnis zur Beistandsform derart korrelieren müssen, dass umso mehr die Beistandsform die in Art. 125 AEUV geschützte Haushaltsdisziplin bedroht, desto enger die Bedingungen gewählt werden müssen. Vorgesehen sind für NGEU nicht rückzahlbare und rückzahlbare Unterstützungen für Investitionsprogramme der Mitgliedstaaten. Um die durch Art. 125 AEUV geschützte Haushaltsdisziplin durch insbesondere nicht rückzahlbare Unterstützungen nicht zu gefährden bedarf es deshalb besonderer Sicherungen. Diese sind indessen vorhanden. Art. 12, Art. 14 sowie Art. 23 Aufbauinstrument-Durchführungs-VO sehen vor, dass die erhaltenen Unterstützungen ausschließlich für Maßnahmen innerhalb der genehmigten Aufbau- und Resilienzpläne verwandt werden dürfen. Eine im Sinne von Art. 125 AEUV schädliche Tilgung von Altschulden darf somit beispielsweise nicht erfolgen. Darüber sowie über die Einhaltung der Aufbau- und Resilienzpläne wacht die Kommission, Art. 10. Sie ist gemäß Art. 22 Abs. 5 Aufbauinstrument-Durchführungs-VO zum Schutz der finanziellen Interessen der Union berechtigt, bei mitgliedstaatlicher Zuwiderhandlung ,die Unterstützung aus der Fazilität anteilig zu kürzen und alle dem Haushalt der Union geschuldeten Beträge einzuziehen bzw. die vorzeitige Rückzahlung des Darlehens zu verlangen“. Überdies kontrolliert die Kommission gemäß Art. 19 jeden gemäß Art. 17 Aufbauinstrument-Durchführungs-VO aufgestellten Aufbau- und Resilienzplan, indem sie diesen bewertet und gegebenenfalls Nachbesserungen einfordert. Insgesamt werden damit mehr als ausreichende Gewährleistungen etabliert, die einerseits die Wahrung des aus Art. 125 AEUV vermittelten Grundsatzes der Haushaltsdisziplin sichern, andererseits aber auch dem Umstand Rechnung tragen, dass im Geiste der Solidarität die Union den Mitgliedstaaten ökonomisch zur Seite springt.

Nach alldem begegnen die finanziellen Unterstützungen an die Mitgliedstaaten auch gemessen an Art. 122 Abs. 2 AEUV keinen durchgreifenden rechtlichen Einwänden. 


\section{Zwischenergebnis}

Es ist damit insgesamt festzuhalten, dass NGEU europarechtlich kompetenzgemäß durch die Union aufgesetzt werden durfte. Art. 311 Abs. 3 und Art. 122 AEUV halten die dafür notwendigen Ermächtigungen bereit.

Eine Abstützung auf beide Normen gebietet sich, weil die Fremdfinanzierung des auf Art. 122 AEUV gestützten Aufbauinstruments in einem unmittelbaren Konnex zu den allgemeinen haushaltsrechtlichen Bestimmungen des Eigenmittelsystems steht, weil insbesondere die als verlorene Zuschüsse konzipierten Programme durch den allgemeinen Haushalt der Union zu refinanzieren sind. Da die Ebene der Kapitalrückführung auf das Engste mit dem Eigenmittelsystem verknüpft ist, muss bereits die Ebene der Kapitalerbringung (die Ermächtigung zur Verschuldung) auf Art. 311 Abs. 3 AEUV und NGEU insgesamt in Verbindung mit der Kapitalverwendung deshalb auf_Art. 122 AEUV gestützt werden.

Das damit einhergehende souveränitätsschonende Verfahren - mitgliedstaatliche Ratifikation notwendig - hat auch legitimatorische Vorteile, die möglicherweise die Suche nach geeigneten Rechtsgrundlagen mit angeleitet haben: Durch die Abstützung der Verschuldungsmöglichkeit auf Art. 311 Abs. 3 AEUV unterstellt sich der Rat dem aus mitgliedstaatlicher Sicht souveränitätsschonendsten Verfahren.

Nach Art. 16 Abs. 3 EUV gilt für Art. 122 AEUV lediglich das Erfordernis einer qualifizierten Mehrheit. Das Verfahren aus Art. 311 Abs. 3 AEUV bindet demgegenüber die Mitgliedstaaten weit intensiver mit ein, weil es nicht nur die konstitutive Zustimmung eines jeden Mitgliedstaats im Rat erfordert, sondern darüber hinaus noch alle Mitgliedstaaten im Einklang mit ihren verfassungsrechtlichen Vorschriften ratifizieren müssen, sodass in etlichen Mitgliedstaaten auch die nationalen Parlamente und nicht nur die Exekutivorgane im Rat befasst werden. ${ }^{50}$ Es entsteht so ein höheres Begründungs- und Öffentlichkeitselement, was die Legitimationsgrundlage des unionalen Handelns stärkt.

Diese Konstruktion birgt freilich auch Risiken.

Als zusätzliche Komplikation im Ringen um eine zukunftsgerichtete Ausgestaltung der Finanzhilfen unter Vorzeichen der Pandemie erwies sich Ende Dezember 2020 der Widerstand Polens und Ungarns. Hintergrund dazu war die Verständigung auf einen EU-Rechtsstaatsmechanismus, der für den Fall anhaltender Rechtsstaatsprobleme in einem Mitgliedstaat ermöglicht, diesem Staat europäische Gelder zu verwehren. ${ }^{51}$ Eingeleitet werden könnte dies mit qualifizierter Mehrheit - also gegen Polen und Ungarn. Da für die Änderung des Eigenmittelbschlusses dem gegenüber Einstimmigkeit erforderlich ist, verkoppelten Polen und Ungarn ihre - erforderliche - Zustimmung für NGEU mit der Forderung nach Abschwächung des Rechtsstaatsmechanismus. Nachdem zwischenzeitlich die Drohung im Raume stand, die Finanzhilfen nur unter 25 Mitgliedstaaten zu vereinbaren, kam es doch noch zum Kompromiss.

50 Zur parlamentarischen Begleitung im Ratifizierungsprozess in einer Vielzahl von Mitgliedstaaten s. https://www.eu2020.de/eu2020-en/news/article/faq-mff-eu2020-maas/2416420.

51 S. die Verordnung über eine allgemeine Konditionalitätsregelung zum Schutz des Haushalts der Union (EU, Euratom) Nr. 2020/2092 des Europäischen Parlaments und des Rates vom 16.12.2020, ABl. L 433 I v. 22.12.2020, 1; zu den möglichen Maßnahmen s. Art. 5, zu den Voraussetzungen Art. 4 und zum Verfahren Art. 6. 
Sehr klar hat dieser Vorfall aber gemacht, dass die wegen Art 311 AEUV erforderliche Einstimmigkeit auch Blockade- und Erpressungspotenzial birgt und dieses Risiko im politischen Handlungskalkül den Vorteilen gegenübersteht. Letztlich überwiegen diese Vorteile deutlich: Gelingt die Verständigung auf einstimmige Zustimmung, dann verbindet sich damit ein erhöhter Legitimationsgehalt, anders als bei Art. 122 AEUV, der lediglich qualifizierte Mehrheit im Rat erfordert.

\section{Verfassungsrechtliches Zustimmungserfordernis nach §3 IntVG iVm. Art. 23 GG - mit einfacher Mehrheit}

Weil sich vorliegend die Frage nach der Unionskompetenz gut beantworten lässt (s. oben) stellt sich die Frage nach der verfassungsrechtlichen Reaktion auf eine europäische Kompetenzüberschreitung („Ultra vires-Akt“) nicht. ${ }^{52}$ Das Unionsrecht verweist in Art. 311 AEUV bei Änderungen des Eigenmittelbeschlusses auf die Zustimmung der Mitgliedstaaten nach ihren jeweiligen verfassungsrechtlichen Vorschriften, was bei herkömmlicher europäischer Sekundärgesetzgebung nicht gefordert ist. Damit eröffnet bereits das Unionsrecht eine (weitere) verfassungsrechtliche Betrachtungsebene.

$\S 3$ IntVG sieht für die Anwendung des Art. 311 AEUV ein Zustimmungsgesetz nach Art. 23 GG vor, eine Regelung die auf das Lissabon-Urteil des Bundesverfassungsgericht zurückgeht. ${ }^{53}$ Das Bundesverfassungsgericht wollte seinerzeit sicherstellen, dass bestimmte Änderungen im Unionsrecht unabhängig von ihrer unionalen Gestalt und Etikettierung in Deutschland wie Vertragsänderungen behandelt werden, mithin ein Zustimmungsgesetz nach Art. 23 GG wie auch sonst bei Änderungen der Verträge verabschiedet wird.

$\mathrm{Ob}$ und wie sich diese Maßgabe in $\S 3$ IntVG auch auf Art. 23 Abs. 1 Satz 3 GG erstreckt, der seinerseits über einen Verweis auf die Verfassungsänderungsbestimmungen des Art. 79 GG für bestimmte Fälle eine Zweidrittelmehrheit anordnet, ist noch immer nicht restlos geklärt. Dies gilt auch für die Abgrenzung von Satz 2 (einfache Mehrheit) und Satz 3 (Zweidrittelmehrheit). Vorliegend ist indessen gleichwohl davon auszugehen, dass eine Zweidrittelmehrheit nicht erforderlich ist.

\section{Allgemeines - die Zweidrittelmehrheit als Ausnahmefall}

Vorab ist an eine recht banale Einsicht zu erinnern, die in der jüngeren Vergangenheit bei der Mitwirkung Deutschlands an der europäischen Integration, immerhin ein Staatsziel unter dem Grundgesetz, etwas aus dem Blick zu geraten droht: Nicht jede überstaatliche Bindung ist eine Hoheitsrechtsübertragung, nicht jede Hoheitsrechtsübertragung erfordert eine verfassungsändernde Mehrheit in den gesetzgebenden Körperschaften.

52 S. in diesem Kontext den Stand der Rechtsprechung des Bundesverfassungsgerichts in BVerfG, Urt. v. 5.5.2020, - PSPP, dazu Mayer, The Ultra Vires Ruling. Deconstructing the Federal Constitutional Court's PSPP decision of 5 May 2020, 16 EuConst 2020, 733.

53 BVerfG 123, 267 (434) - Lissabon. 
Nicht zuletzt durch eine überschießende Zustimmungsgesetzpraxis erscheinen mittlerweile Zweidrittelmehrheiten in manchen Diskussionen nicht selten fast schon als der gewohnheitsrechtlich anzunehmende Normalfall europabezogener Zustimmungsoder Vertragsgesetze des Parlaments. Dies trifft indessen nicht zu.

Hier ist es hilfreich, sich über bestimmte Grundannahmen zu vergewissern. Demokratie ist Mehrheitsherrschaft. Qualifizierte Mehrheitserfordernisse durchbrechen diese Grundannahme, indem einer Minderheit Blockademöglichkeiten gegeben werden, die bei strikter Geltung des Mehrheitsprinzips nicht bestünden. Sie sind daher rechtfertigungsbedürftig und grundsätzlich die Ausnahme.

Die simpelste Erklärung für die gesonderten Regeln mit erhöhten Quoren zur Änderung des Verfassungsgesetzes geht davon aus, dass in der Verfassung grundsätzliche Entscheidungen niedergelegt sind, die dem Hin und Her des gewöhnlichen politischen Prozesses mit seinen wechselnden Mehrheiten entzogen sind. Dies betrifft systemische und substantielle Grundentscheidungen, die nicht mit jedem Wechsel der einfachen Mehrheit zur Disposition stehen sollen. Nur bei besonderen Entwicklungen tritt der politische Prozess gleichsam aus der herkömmlichen Spur und es werden entsprechend besondere Mehrheiten erforderlich, um diese Verfassungsmomente auch in der Verfassungsurkunde dauerhaft festzuschreiben. ${ }^{54}$

Dies bedeutet allerdings nicht, dass automatisch alle wichtigen oder über eine Legislaturperiode hinauswirkenden Entscheidungen nach der Verfassunggebung von 1949 mit Zweidrittelmehrheit beschlossen worden wären.

Etliche die Bundesrepublik prägende politische Entscheidungen, die nicht selten auch tiefgreifende strukturelle Bedeutung hatten und haben, sind mit einfacher Mehrheit beschlossen worden. Beispiele reichen vom Beitritt der Bundesrepublik zur EMRK und zur UNO, Auslandseinsätzen der Bundeswehr, Grundentscheidungen bei den Renten- und Sozialsystemen bis zur Einführung der gleichgeschlechtlichen Ehe.

Hier ist letztlich die Grundaussage des Art. 79 Abs. 1 Satz 1 GG maßgeblich, der anders als noch die Weimarer Reichsverfassung eine nicht explizit den Text ändernde Verfassungsänderung ausschließt. Die Frage ist dann schlicht, ob eine Verfassungsänderung erforderlich ist, oder ein einfaches Gesetz genügt. Die tatsächliche oder gefühlte Wichtigkeit einer Frage ist damit kein zentrales Kriterium.

Das Zweidrittelerfordernis des Art. 23 Abs. 1 Satz 3 GG stellt sich als Durchbrechung des Grundsatzes des Art. 79 Abs. 1 Satz 1 GG für den speziellen Fall des Unionsrechts oder des Unionsrechtsbezugs und damit als Sonderfall dar, der auch Sonderkonstellationen vorbehalten bleiben muss.

\section{Voraussetzungen für die Anwendung des Art. 23 Abs. 1 Satz 2 GG (einfache Mehrheit)}

Art. 23 Abs. 1 Satz 3 GG verweist auf die Bestimmungen zur Verfassungsänderung in Art. 79 Abs. 2 und 3 GG und ermöglicht der Sache nach materielle „Verfassungsände-

$54 \mathrm{Zu}$ den „constitutional moments“ als theroretischer Ansatz, mit dem versucht wird, diese Beobachtung einzuordnen Ackerman, We the people: Foundations, 1991. 
rungen ohne Verfassungstextänderungen“. ${ }^{55}$ Unter welchen Voraussetzungen eine Hoheitsrechtsübertragung im Kontext des Art. 23 GG eine Zweidrittelmehrheit erfordert, ist dabei nicht abschließend geklärt. Das Bundesverfassungsgericht hat hierzu jüngst geäußert, dass „die im Schrifttum vertretenen Meinungen zum Verhältnis von Art. 23 Abs. 1 Satz 2 und Satz 3 GG in kaum mehr überschaubare Verästelungen zerfallen". ${ }^{56}$ Dies liegt nicht zuletzt daran, dass in der bisherigen Verfassungspraxis häufig die Mehrheiten bei der Aktivierung des Art. 23 GG ohnehin das Zweidrittelquorum überschritten haben, sodass es auf eine genaue Bestimmung des Mehrheitserfordernisses nicht ankam. ${ }^{57}$

Hierbei hat immer wieder auch eine Rolle gespielt, dass im föderalen Gefüge nicht selten in Bundestag und Bundesrat gegenläufige Mehrheiten bestehen, so dass die Minderheit im Bundestag über eine Mehrheit im Bundesrat ein Zweidrittelquorum aus politischen Gründen erzwingen konnte, ohne dass die Voraussetzungen des Art. 23 Abs. 1 Satz 3 GG im Detail geprüft worden wären. Von einem verfassungsgewohnheitsrechtlichen Zweidrittelquorum bei der Übertragung von Hoheitsrechten kann gleichwohl keine Rede sein. Dafür bestehen noch immer zu viele Fälle, in denen Hoheitsrechte mit einfacher Mehrheit übertragen werden.

Eine Durchsicht der Gesetzgebungspraxis seit 2009 ergibt Folgendes:

- Ein Zustimmungsgesetz auf der Grundlage von Art. 23 GG ist am häufigsten im Hinblick auf die Anwendung des Art. 352 AEUV verabschiedet worden. Dies geht ebenfalls auf eine Vorgabe des Bundesverfassungsgerichts aus dem Lissabon-Urteil zurück, die in $\S 8$ IntVG niedergelegt ist. In der Begründung wurde jeweils auf Art. 23 Abs. 1 GG und $\S 8$ IntVG Bezug genommen, alle Entwürfe wurden mit der Einleitungsformel für einfache Mehrheit vorgelegt und auch so beschlossen. ${ }^{58}$

- Bei der SSM-Verordnung (Kontext Bankenunion) stellte der Gesetzentwurf der Bundesregierung auf Art. 23 Abs. 1 Satz 2 ab, obwohl Art. 127 Abs. 6 AEUV weder im Lissabon-Urteil noch im IntVG als ein Fall einer unzureichenden Kompetenzübertragung bezeichnet wurde. ${ }^{59}$

55 Lorz/Sauer, Verfassungsändernde Mehrheiten für die Stabilisierung des Euro?, EuR 2012, S. $682(685)$.

56 BVerfG, 2 BvR 739/17, Beschl. v. 13.2.2020 - EPGÜ, Rn. 129.

57 Näher dazu Wollenschläger, in: Dreier, Grundgesetz Kommentar, Bd. II, 3. Aufl. 2015, Art. 23 GG Rn. 55, 58; Wendel, Permeabilität im europäischen Verfassungsrecht, 2011, S. 240 f.; s. auch die seinerzeitige Diskussion in Bundestag und Bundesrat über das Zustimmungsgesetz zum Vertrag von Nizza als Fall des Art. 23 Abs. 1 Satz 3 GG, Gesetzesentwurf der Bundesregierung vom 5. Mai 2001, BT-Drs. 14/6146 S. 6 und 57 gegenüber 55.

58 Einige Beispiele: EU-Grundrechteagentur, Mehrjahresrahmen, BT-Drs. 17/10760, BGB1. II 2012, 1378; Aufhebung des Beschlusses 2007/124/EG (Euratom), BT-Drs. 18/824, BGBl. II 2014, 410; Programm „Pericles“, BT-Drs. 18/1225, BGBl. II 2014, 426; Historische Archive der EU, BT-Drs. 18/1779, BGB1. II 2014, 1010; Sozialgipfel Wachstum und Beschäftigung, BT-Drs. 18/2953, BGBl. II 2015, 15; Standpunkte Union Albanien und Serbien, BT-Drs. 18/9990, BGB1. II 2017, 298; Anwendung Wettbewerbsrecht Kanada-EU, BT-Drs. 18/10808, BGBl. II 2017, 498; EU-Grundrechteagentur, BT-Drs. 18/12332, BGBl. II 2017, 810 .

59 BT-Drs. 17/13470 und BT-Drs. 17/13829, BGB1. II 2013, 1050. 
- Beim einheitlichen Abwicklungsfonds (ebenfalls Bankenunion) ging es um einen völkerrechtlichen Vertrag in Ergänzung einer EU-Verordnung, die Begründung bezog sich auf Art. 59 Abs. 1 iVm. Art. 23 Abs. 1 Satz 2 GG. ${ }^{60}$

- Bei der Änderung der ESM-Finanzhilfeinstrumente ist ein vorheriges Zustimmungsgesetz nach Art. 23 GG im ESM-Errichtungsgesetz vorgesehen. ${ }^{61}$

Eine Zweidrittelmehrheit hat nur in zwei Fällen überhaupt eine Rolle gespielt:

- Das Zustimmungsgesetz nach Art. 23 GG zum ESM-Vertrag wurde in den Ausschussberatungen auf eine Einleitungsformel auf Einhaltung der Zweidrittelmehrheit umgestellt, ohne dies näher zu begründen. ${ }^{62}$

- Das Vertragsgesetz zum Fiskalvertrag sah unter Bezugnahme auf Art. 23 Abs. 1 Satz 3 GG eine Zweidrittelmehrheit vor, weil die Verträge über die EU geändert wurden und die Änderung darauf abzielt, dass Deutschland auf Verfassungsebene an die Schuldenbegrenzung/-vermeidung gebunden ist. ${ }^{63}$

Grundsätzlich sind verfassungsändernde Mehrheiten, die das Grundgesetz nicht erfordert, ohne Bedeutung: der verfassungsrechtliche Prüfungsmaßstab kann nicht durch das Verabschieden eines Gesetzes mit verfassungsändernden Mehrheiten verändert werden. ${ }^{64}$ Trotz der teilweise konträren Positionen zu Art. 23 Abs. 1 Satz 2 und 3 GG ergibt sich aus dem Wortlaut der Verfassung (dazu a.) und dem im Grundgesetz angelegten Konzept der Hoheitsrechtsübertragung (dazu b. bis f.) hinreichend deutlich, dass die Zustimmung zum NGEU-Paket keine Zweidrittelmehrheit erfordert (g.).

\section{a) Wortlaut und Genese}

Eine Zweidrittelmehrheit gemäß Art. 23 Abs. 1 Satz 3 GG ist nach dem Wortlaut des Grundgesetzes dann erforderlich, wenn zwei Voraussetzungen kumulativ vorliegen: Erstens muss es in der Sache um die Begründung der Europäischen Union, Änderungen ihrer vertraglichen Grundlagen oder vergleichbare Regelungen gehen und zweitens müssen diese Regelungen Verfassungsrelevanz insofern aufweisen, als dass das „Grundgesetz seinem Inhalt nach geändert oder ergänzt wird oder solche Änderungen oder Ergänzungen ermöglicht werden“".

In der Vorläufer- und Parallelbestimmung zu Art. 23 GG, Art. 24 GG, findet sich eine dem Art. 23 Abs. 1 Satz 3 GG vergleichbare Qualifikation nicht. Im Parlamentarischen Rat war seinerzeit ein Antrag, die Übertragung von Hoheitsrechten nur mit den Mehrheiten des Art. 79 Abs. 2 GG zuzulassen, abgelehnt worden, um die Eingliederung Deutschlands in internationale Kooperationssysteme ganz bewusst zu erleichtern. ${ }^{65}$ Damit ist aber auch dokumentiert, dass von Anfang ein Zweidrittelquorum als

60 BT-Drs. 18/2576, BGBl. II 2014, 1298.

$61 \S 2$ Abs. 2, Ersatz für fehlenden Ratifizierungsvorbehalt; BT-Drs. 18/2580, BGB1. I 2014, 1821.

62 BT-Drs. 17/9045 und 17/10126, BGBl. 2012 II S. 981.

63 BT-Drs. 17/9046, BGB1 2012 II, 1006.

64 So zutreffend Lorz/Sauer, Verfassungsändernde Mehrheiten für die Stabilisierung des Euro?, EuR 2012, S. 682 (695).

65 Tomuschat, in: Bonner Kommentar zum Grundgesetz, 42. EL September 1981, Art. 24 GG Rn. 33. 
Hindernis für die europäische und internationale Zusammenarbeit Deutschlands eingeordnet wurde. Art. 23 GG ist demgegenüber eine Weiterentwicklung der Verfassung, zugleich hat aber gerade Abs. 1 Satz 3 auch Kompromisscharakter, da über die Reichweite der verfassungsändernden Mehrheitsschwelle innerhalb der Gemeinsamen Verfassungskommission nach der Wiedervereinigung, auf die Art. 23 GG zurück geht, durchaus intensiv gestritten wurde. ${ }^{66}$ Dabei konnte sich der Vorschlag, für Art. 23 Abs. 1 Satz 3 GG das Erfordernis des „Eingriffs in die wesentlichen Strukturen des Grundgesetzes" vorzusehen, in der Gemeinsamen Verfassungskommission nicht durchsetzen. ${ }^{67}$ Dass die Ergänzung insbesondere im Hinblick auf Abgrenzungsschwierigkeiten beim Begriff der „,wesentlichen Strukturen“ verworfen wurde, ${ }^{68}$ macht den Kompromisscharakter der in das Grundgesetz aufgenommenen Vorschrift deutlich. Diesen Kompromisscharakter gilt es, im Blick zu behalten.

Zugleich ergibt sich hier bereits ein Indiz dafür, dass es eben doch um qualifizierte Hoheitsrechtsübertragungen gehen soll, die auf einen abstrakt-generellen und zugleich eindeutigen und anschaulichen Begriff zu bringen dem verfassungsändernden Gesetzgeber 1993 schlicht nicht gelungen ist.

\section{b) Hoheitsrechtsübertragung}

Ganz überwiegend wird vertreten, dass Art. 23 Abs. 1 Satz 3 GG eine Hoheitsrechtsübertragung im Sinne des Art. 23 Abs. 1 Satz 2 GG voraussetzt. ${ }^{69}$ Dabei wird der Begriff der Hoheitsrechtsübertragung überwiegend weit ausgelegt.

\section{c) Unterscheidung zwischen einfacher und qualifizierter Übertragung von Ho- heitsrechten}

Teile der Literatur fordern, dass jegliche Hoheitsrechtsübertragung im Sinne des Art. 23 Abs. 1 Satz 2 GG auch dem Erfordernis einer Zweidrittelmehrheit des Satz 3 unterliegen soll. ${ }^{70}$ Gegen diese Sicht spricht indessen, dass mit der Begründung der Europäischen Union, der Änderungen ihrer vertraglichen Grundlagen und vergleich-

66 Näher dazu Wendel, Permeabilität im europäischen Verfassungsrecht, 2011, S. 243.

67 Vgl. Scholz, in: Maunz/Dürig, Grundgesetz-Kommentar, 56. EL Dezember 2009, Art. 23 GG Rn. 117 unter Verweis auf den Bericht der Gemeinsamen Verfassungskommission vom 5. November 1993, BT-Drs. 12/6000, S. 21.

68 Bericht der Gemeinsamen Verfassungskommission vom 5. November 1993, BT-Drs. 12/6000, S. 21.

69 Vgl. Wollenschläger, in: Dreier, Grundgesetz Kommentar, Bd. II, 3. Aufl. 2015, Art. 23 GG Rn. 53 f. mwN.; König, Die Übertragung von Hoheitsrechten im Rahmen des europäischen Integrationsprozesses, 2000, S. 314 ff.; a.A. Schorkopf, in: Bonner Kommentar zum Grundgesetz, 153. EL September 2011, Art. 23 Rn. 81, der Art. 23 Ab. 1 Satz 3 GG in bestimmten Konstellationen auch unabhängig von Satz 2 anwenden will.

70 Streinz, in: Sachs, Grundgesetz, 7. Aufl. 2014, Art. 23 Rn. 72 f. mwN.; Schorkopf, in: Bonner Kommentar zum Grundgesetz, 153. EL September 2011, Art 23. Rn. 81; UerpmannWittzack, in: v. Münch/Kunig, Grundgesetz-Kommentar, Bd. I, 6. Aufl. 2012, Art. 23 GG Rn. 45, 52; Baach, Parlamentarische Mitwirkung in Angelegenheiten der Europäischen Union, 2008, S. $118 \mathrm{ff}$. 
barer Regelungen bestimmte besondere Hoheitsrechtsübertragungen hervorgehoben werden. Hätte man alle Hoheitsrechtsübertragungen dem Zweidrittelquorum unterstellen wollen, dann hätte man Satz 3 schlicht an Satz 1 anschließen können. Daraus ergibt sich im Umkehrschluss, dass es neben den qualifizierten Hoheitsrechtsübertragungen des Satz 3 auch „einfache“ Hoheitsrechtsübertragungen im Sinne des Satz 2 ohne Zweidrittelerfordernis - geben muss. Andernfalls würde der Differenzierung im Wortlaut des Art. 23 Abs. 1 GG nicht Rechnung getragen. ${ }^{71}$ Insoweit stehen die Tatbestände von Satz 2 und Satz 3 nebeneinander, die Tatbestandsvoraussetzungen sind jeweils gesondert zu prüfen. ${ }^{72}$ Satz 3 setzt dabei Satz 2 voraus, erfasst aber nur eine Teilmenge von den in Satz 2 adressierten Fällen. Auch das Bundesverfassungsgericht unterscheidet nach wie vor zwischen Art. 23 Abs. 1 Satz 2 und Art. 23 Abs. 1 Satz 3 GG. ${ }^{73}$

\section{d) Abgrenzung zwischen Art. 23 Abs. 1 Satz 2 und Satz 3 GG}

Fraglich ist damit gleichwohl, wie die Trennlinie zwischen einfacher und qualifizierter Übertragung von Hoheitsrechten zu ziehen ist. In der Literatur wird richtigerweise vorgeschlagen, für die Anwendbarkeit des Satz 3 nach der Tragweite der Hoheitsrechtsübertragung oder Vertragsänderung zu unterscheiden. Es reicht insofern nicht jede Verfassungsrelevanz, vielmehr wird eine strukturelle Verfassungsrelevanz gefordert. ${ }^{74}$ In der amtlichen Begründung zur Grundgesetzänderung wird dazu 1992 Folgendes ausgeführt:

„Satz 3 schreibt für die Schaffung von neuem primärem Gemeinschaftsrecht - einschließlich der Gründung der Europäischen Union - unter bestimmten Voraussetzungen verfassungsändernde Mehrheiten in Bundestag und Bundesrat vor, wenn dadurch das Grundgesetz seinem Inhalt nach geändert oder ergänzt wird oder solche Änderungen oder Ergänzungen ermöglicht werden.

Geht man mit dem Bundesverfassungsgericht davon aus, dass letztlich jede Hoheitsrechtsübertragung eine materielle Verfassungsänderung bedeutet, weil jedenfalls in die verfassungsrechtlich festgelegte Zuständigkeitsordnung eingegriffen wird (vgl. BVerfGE 58, 1, 36), stellt sich die Frage, in welchen Fällen der einfache Gesetzgeber von der Übertragungsermächtigung nach Satz 2 Gebrauch machen

71 Scholz, in: Maunz/Dürig, Grundgesetz-Kommentar, 56. EL Dezember 2009, Art. 23 Rn. 118.

72 So auch Scholz, in: Maunz/Dürig, Grundgesetz-Kommentar, 56. EL Dezember 2009, Art. 23 Rn. 118,. Siehe auch Heyde, in: Umbach/Clemens, GG-Kommentar, 2002, Art. 23 Rn. 57 ff. mwN.; Wollenschläger, Völkerrechtliche Flankierung des EU-Integrationsprogramms als Herausforderung für den Europa-Artikel des Grundgesetzes (Art. 23 GG), NVwZ 2012, S. 713 (715), mwN.

73 BVerfGE 123, 267 (387, Rn. 312; 391, Rn. 319; 434, Rn. 412) - Lissabon; jüngst auch in BVerfG, 2 BvR 739/17, Beschl. v. 13.2.2020 - EPGÜ, Rn. 129.

74 Jarass, in: Grundgesetz für die Bundesrepublik Deutschland. Kommentar, 14. Aufl. 2016, Art. 23 Rn. 36, 38. Siehe auch Pernice, in: Dreier, Grundgesetz Kommentar, Bd. II, 2. Aufl. 2006, Art. 23 Rn. 90; Wollenschläger, in: Dreier, Grundgesetz Kommentar, Bd. II, 3. Aufl. 2015, Art. 23 Rn. 57. 
kann. Anwendungsfälle für die Hoheitsrechtsübertragung nach Satz 2 könnten sich dann ergeben, wenn Änderungen des Unions-Vertrages zu ratifizieren sind, die von ihrem Gewicht her der Gründung der Europäischen Union nicht vergleichbar sind und insoweit nicht die ,, Geschäftsgrundlage" dieses Vertrages betreffen. “

Und weiter:

„In seinem Anwendungsbereich erlaubt Satz 3 - in den Grenzen des Artikels 79 Abs. 3 GG - materielle Verfassungsänderungen im vorstehend erwähnten Sinne sowie die Anordnung des innerstaatlichen Anwendungsvorrangs für Recht der Europäischen Union, das mit Vorschriften des Grundgesetzes kollidiert, unter Befreiung vom Textänderungsgebot des Artikels 79 Abs. 1 Satz 1 GG. Andererseits verlangt er im Unterschied zu Artikel 24 Abs. 1 GG und der Regelung in Satz 2 ausnahmslos die Mehrheiten des Artikels 79 Abs. 2 GG und stellt insoweit eine Einschränkung der Übertragungsermächtigung für den einfachen Gesetzgeber dar, wie sie durch die Solange-Rechtsprechung des Bundesverfassungsgerichts zu Artikel 24 Abs. 1 GG definiert wird (vgl. BVerfGE 73, 337, $375 f$.). Die dadurch möglicherweise eintretenden, verfassungspolitisch an sich unerwünschten Verluste an Verfassungsklarheit dürften allerdings auch bei Geltung des Textänderungsgebots schwer zu vermeiden sein. Denn die jeweilige Anpassung des Grundgesetzes an die durch Gemeinschaftsrecht gestaltete Rechtslage wird häufig nicht so einfach zu formulieren sein wie die in Artikel 1 Nr. 3 und 4 dieses Entwurfs vorgeschlagenen Grundgesetzänderungen. Auch ist die Bedeutung gemeinschaftsrechtlich veranlasster Grundgesetzänderungen — wie die soeben erwähnten Beispiele zeigen ohne Kenntnis des Gemeinschaftsrechts nicht zu erschließen. "75

Ein maßgeblicher Gesichtspunkt ist danach, ob es um einen Vorgang geht, der vom Gewicht her der Gründung der Europäischen Union vergleichbar ist und insoweit die „Geschäftsgrundlage“ des Primärrechts betrifft. Zugleich muss sich der Vorgang als materielle Verfassungsänderung darstellen, und zwar ohne dass es dafür eines weiteren Zustimmungsaktes bedarf. Dabei ist nochmals zu betonen, dass nicht alles, was politisch besonders bedeutsam ist oder erscheint, verfassungsändernden Charakter hat. $^{76}$

Das Bundesverfassungsgericht hat jüngst in seinem mit einer knappen 5 zu 3 Mehrheit ergangenen Beschluss zum Europäischen Patentgerichtsübereinkommen (EPGÜ) ${ }^{77}$ einige weitere Hinweise zur Abgrenzung der Sätze 2 und 3 gegeben: Dass Änderungen des Grundgesetzes im Sinne von Art. 23 Abs. 1 Satz 3 GG „ermöglicht“ werden sei ,vor allem der Fall, wenn das Integrationsgesetz und/oder der völkerrechtliche Vertrag - als innerstaatliches Recht konzipiert - eine ausschließliche Zuständigkeit der Europäischen Union begründet oder eine vollständige Verdrängung des Bundesgesetzgebers ermöglicht (Art. 73 f., 105 GG), Eingriffe in die Gesetzgebungszuständigkeit der Länder zugelassen (Art. 30, 70 GG) oder die Verwaltungs- (Art. 83 ff.,

75 Gesetzesentwurf der Bundesregierung, Entwurf eines Gesetzes zur Änderung des Grundgesetzes vom 2. Oktober 1992, BT-Drs. 12/3338, S. 7.

76 Lorz/Sauer, Verfassungsändernde Mehrheiten für die Stabilisierung des Euro?, EuR 2012, S. $682(691)$.

77 BVerfG, 2 BvR 739/17, Beschl. v. 13.2.2020 - EPGÜ. 
108 GG) und Rechtsprechungskompetenzen (Art. 92 GG) von Bund und Ländern beeinträchtigt werden. Eine Europäisierung grundgesetzlicher Vorgaben ist ferner anzunehmen, wenn das Integrationsgesetz und/oder der völkerrechtliche Vertrag die verfassungsrechtlichen Vorgaben für die kommunale Selbstverwaltung (Art. 28 Abs. 2 GG), die Bundesbank (Art. 88 GG) oder den Gerichtsaufbau (Art. 92 ff., 96 GG) verändert oder überformt. “78

Dagegen stellen vor allem im Integrationsprogramm hinreichend bestimmt angelegte und mit einer Zweidrittelmehrheit bereits gebilligte Übertragungen keine (abermalige) materielle Änderung des Grundgesetzes dar. Auf sie soll allein Art. 23 Abs. 1 Satz 2 GG Anwendung finden. ${ }^{79}$

\section{e) Sonderkonstellation Zustimmungsgesetz nach $§ 3$ IntVG?}

Die Dinge werden vorliegend noch weiter verkompliziert, weil die Anwendung des Art. 23 GG durch $\S 3$ IntVG gleichsam angeordnet wird. Das IntVG setzt die Vorgaben des Lissabon-Urteils des Bundesverfassungsgerichts um. In diesem Urteil hatte das Bundesverfassungsgericht eine Reihe von Vertragsbestimmungen identifiziert, die nach seiner Auffassung als verdeckte Vertragsänderungen bei Aktivierung eine erneute Ratifikation in Deutschland erfordern. ${ }^{80}$ Darauf, ob tatsächlich eine Hoheitsrechtsübertragung erfolgt, kommt es nach dem Wortlaut des IntVG - im Anschluss an die Vorgabe aus dem Lissabon-Urteil - gar nicht an. Das IntVG verlangt ein Gesetz nach Art. 23 Abs. 1 GG unabhängig von einer Hoheitsrechtsübertragung. Es besteht mit $\S 3$ Abs. 1 IntVG gewissermaßen eine Regelvermutung, dass bei Aktivierung von Art. 311 AEUV eine Hoheitsrechtsübertragung erfolgt.

Dass die Regelvermutungen des IntVG auch daneben liegen können, zeigt aber die Anwendungspraxis des $\S 8$ IntVG, der bei Aktivierung des Art. 352 AEUV ein Zustimmungsgesetz vorsieht: Dies hat zu der skurrilen Situation geführt, dass sogar unverbindliche Akte der EU in Deutschland eine Ratifikationsgesetzgebung ausgelöst haben, nur weil diese auf Art. 352 AEUV gestützt waren. ${ }^{81}$

78 BVerfG, 2 BvR 739/17, Beschl. v. 13.2.2020 - EPGÜ, Rn. 130.

79 BVerfG, 2 BvR 739/17, Beschl. v. 13.2.2020 - EPGÜ, Rn. 129.

80 BVerfGE 123, 267 (387) - Lissabon: „Den Verträgen werden durch den Vertrag von Lissabon weitere Bestimmungen eingefügt, die Art. 48 Abs. 6 EUV-Lissabon nachgebildet sind, aber auf einen bestimmten Sachbereich begrenzt und durch den Vertrag von Lissabon erweitert werden (vgl. Art. 42 Abs. 2 UAbs. 1 EUV-Lissabon - Einführung einer Gemeinsamen Verteidigung; Art. 25 Abs. 2 AEUV - Erweiterung der Rechte der Unionsbürger; Art. 218 Abs. 8 UAbs. 2 Satz 2 AEUV - Beitritt der Europäischen Union zur EMRK; Art. 223 Abs. 1 UAbs. 2 AEUV - Einführung eines einheitlichen Wahlverfahrens zum Europäischen Parlament; Art. 262 AEUV - Zuständigkeit der Europäischen Union zur Schaffung europäischer Rechtstitel für geistiges Eigentum; Art. $311 \mathrm{Abs} .3 \mathrm{AEUV}-$ Festlegung der Eigenmittel der Europäischen Union). “ [Hervorhebung hinzugefügt].

81 Gesetz zum Vorschlag für eine Empfehlung des Rates zum Zugang zum Sozialschutz für Arbeitnehmer und Selbstständige, BGBl. 2019 II 444. 


\section{f) Zwischenergebnis}

Festzuhalten ist, dass nicht jeder Anwendungsfall des IntVG auch im Konkreten eine Hoheitsrechtsübertragung zum Gegenstand hat, ferner, dass nicht jede Hoheitsrechtsübertragung eine Zweidrittelmehrheit erfordert.

Jedenfalls die im Integrationsprogramm hinreichend bestimmt angelegten und mit einer Zweidrittelmehrheit bereits gebilligten Übertragungen stellen keine (abermalige) materielle Änderung des Grundgesetzes dar. Auf sie ist jedenfalls allein Art. 23 Abs. 1 Satz 2 GG anzuwenden.

\section{g) NGEU und die Voraussetzungen für die Anwendung des Art. 23 Abs. 1 Satz 3 GG}

Das Zustimmungsgesetz zu NGEU ist nach alledem kein Anwendungsfall des Art. 23 Abs. 1 Satz 3 GG. Eine Zweidrittelmehrheit ist bei der Beschlussfassung nicht erforderlich. Weder wird eine ausschließliche Zuständigkeit der EU begründet oder eine vollständige Verdrängung des Bundesgesetzgebers ermöglicht, noch werden Eingriffe in die Gesetzgebungszuständigkeit der Länder zugelassen oder die Verwaltungs- und Rechtsprechungskompetenzen von Bund und Ländern beeinträchtigt. Auch die verfassungsrechtlichen Vorgaben für die kommunale Selbstverwaltung, die Bundesbank oder den Gerichtsaufbau bleiben unberührt. Art. 122 AEUV, der ausdrücklich an die Mitgliedstaaten appelliert, in Notlagen „,im Geiste der Solidarität“ zu agieren und Art. 311 AEUV werden nicht geändert, sondern betätigt. Die NGEU-Maßnahmen stellen sich damit als im Integrationsprogramm in Gestalt von Art. 122 AEUV iVm. Abs. 311 hinreichend bestimmt angelegte und mit einer Zweidrittelmehrheit bereits gebilligte Übertragungen dar. Es findet alleine Art. 23 Abs. 1 Satz 2 GG Anwendung.

\section{Keine verfassungsrechtlichen Hindernisse aus der Budgetverantwortung des Bundestages}

Neben der Kompetenzfrage und der Zustimmungsgesetzfrage ist möglicherweise auch die haushaltsverfassungsrechtliche Tragweite von NGEU für die nationale verfassungsrechtliche Einordnung von Belang. Das Bundesverfassungsgericht hat bereits im Lissabon-Urteil 2009 zur Budgetverantwortung des Bundestages einige allgemeine, perspektivische Hinweise gegeben. Wörtlich heißt es:

„,Eine das Demokratieprinzip und das Wahlrecht zum Deutschen Bundestag in seinem substantiellen Bestimmungsgehalt verletzende Übertragung des Budgetrechts des Bundestages läge vor, wenn die Festlegung über Art und Höhe der den Bürger treffenden Abgaben in wesentlichem Umfang supranationalisiert würde. Der Deutsche Bundestag muss dem Volk gegenüber verantwortlich über die Summe der Belastungen der Bürger entscheiden. Entsprechendes gilt für wesentliche Ausgaben des Staates. In diesem Bereich obliegt gerade die sozialpolitische Verantwortung dem demokratischen Entscheidungsprozess, auf den die Bürger mit der freien und gleichen Wahl einwirken wollen. Die Hoheit über den Haushalt ist der Ort konzep- 
tioneller politischer Entscheidungen über den Zusammenhang von wirtschaftichen Belastungen und staatlich gewährten Vergünstigungen. Deshalb wird die parlamentarische Aussprache über den Haushalt - einschließlich des Maßes der Verschuldung - als politische Generaldebatte verstanden. Nicht jede haushaltswirksame europäische oder internationale Verpflichtung gefährdet die Gestaltungsfähigkeit des Bundestages als Haushaltsgesetzgeber." 82

In der EFSF-Entscheidung hat das Bundesverfassungsgericht diese Vorgaben weiter präzisiert:

„,Danach läge eine das Demokratieprinzip und das Wahlrecht zum Deutschen Bundestag verletzende Übertragung wesentlicher Bestandteile des Budgetrechts des Bundestages jedenfalls dann vor, wenn die Festlegung über Art und Höhe der den Bürger treffenden Abgaben in wesentlichem Umfang supranationalisiert und damit der Dispositionsbefugnis des Bundestages entzogen würde (vgl. BVerfGE 123, 267 $<361>$ ). " 83

An diesen Maßstäben gemessen begegnet das NGEU-Programm keinen verfassungsrechtlichen Bedenken.

Zunächst ist klarzustellen, dass Unterstellungen von Haftungsrisiken für den Bundeshaushalt in dreistelliger Milliardenhöhe völlig haltlos sind. Zur Deckelung des jährlichen Finanzbedarfs aus NGEU besteht bereits eine Sicherung auf Ebene des Schulden- und Liquiditätsmanagements. Zwecks stetiger und vorhersehbarer Verringerung der Verbindlichkeiten ${ }^{84}$ darf der Betrag für die Rückzahlung des Kapitalbetrags für nicht rückzahlbare Unterstützungen jährlich 7,5\% des Höchstbetrags für solche Unterstützungen nicht übersteigen. ${ }^{85}$ In Rede stehen damit 7,5\% von 390 Mrd. $€{ }^{86}$ also 29,25 Mrd. € pro Jahr. Diese werden aus dem EU-Haushalt erbracht. ${ }^{87}$

Zwar enthält der Eigenmittelbeschluss eine Nachschusspflicht zulasten der Mitgliedstaaten in Art. 9 Abs. 5 Eigenmittelbeschluss, wenn der Finanzbedarf die Haushaltsmittel der Union übersteigt. Dabei kann ein Mitgliedstaat sogar mit einem höheren Anteil belegt werden, wenn ein oder mehrere andere Mitglieder dem Kapitalabruf nicht nachkommen können. ${ }^{88}$ Von einer Belastung in einem die Finanzkraft der Mitgliedstaaten überfordernden Umfang kann aber auch dann nicht die Rede sein, weil Art. 9 Abs. 6 Eigenmittelbeschluss für die jährliche Inanspruchnahme eines Mitgliedstaates eine Grenze setzt. Es kann danach für einen Mitgliedstat maximal sein BNEgestützter relativer Anteil gemäß Art. 6 Eigenmittelbeschluss i.H.v. 0,6 Prozentpunkten des BNE eingefordert werden. Selbst in Verbindung mit der im Vergleich zur vorherigen Finanzperiode 2014-20 von 1,23 auf 1,4\% erhöhten Eigenmittelobergrenze würde auf Deutschland eine maximale zusätzliche Belastung i.H.v. 27,92 Mrd. € bzw.

82 BVerfGE 123, 267, Rn. 265 - Lissabon. [Hervorhebungen hinzugefügt].

83 BVerfG 129, 124, Rn. 126 - EFSF. [Hervorhebungen hinzugefügt].

84 21. Erwägungsgrund Eigenmittelbeschluss.

85 Art. 5 Abs. 2 UAbs. 3 Eigenmittelbeschluss.

86 Art. 5 Abs. 2 UAbs. 3 iVm. Abs. 1 UAbs. 1 lit. b Eigenmittelbeschluss.

87 Art. 9 Abs. 5 UAbs. 1 Eigenmittelbeschluss.

88 Art. 9 Abs. 5 UAbs. 2 Eigenmittelbeschluss. 
lediglich 21,75 Mrd. $€$ für NGEU entfallen. Beides würde bei einem derzeitigen Bundeshaushalt von 360 Mrd. $€$ immer noch nicht ausreichen, um ernsthaft einen solchen Umfang annehmen zu können, dass dem Haushaltsgesetzgeber kein wesentlicher Gestaltungsraum bleiben würde.

Dies gilt selbst in einem absurden Extremszenario, in dem alle anderen Mitgliedstaaten vollständig ausfallen und die Rückzahlungen gemäß dem o.g. Maximalbetrag ganz alleine von Deutschland erbracht würden und in dem so die Gesamtsumme von 750 Mrd. € nach 27 Jahren (Zinsen außer Betracht) zurückgezahlt wäre.

Noch einmal das Bundesverfassungsgericht in Wortlaut:

„Eine unmittelbar aus dem Demokratieprinzip folgende Obergrenze für die Übernahme von Gewährleistungen könnte nur überschritten sein, wenn sich im Eintrittsfall die Gewährleistungen so auswirkten, dass die Haushaltsautonomie jedenfalls für einen nennenswerten Zeitraum nicht nur eingeschränkt würde, sondern praktisch vollständig leerliefe. " 89

Dies ist vorliegend ersichtlich nicht der Fall.

\section{Parallele zur Beschlussfassung über das Haushaltsgesetz}

Das Bundesverfassungsgericht hat im Übrigen auf die Parallele zwischen der Beschlussfassung über den Bundeshaushalt - mit einfacher Mehrheit - und dem europäischen Verfahren hingewiesen. Wörtlich heißt es:

„,Die haushaltspolitische Gesamtverantwortung wird grundsätzlich durch Verhandlung und Beschlussfassung im Plenum wahrgenommen, durch den Beschluss über das Haushaltsgesetz, durch finanzwirksame Gesetze oder durch sonstige konstitutive Beschlüsse des Bundestages (vgl. BVerfGE 130, $318<347>$ ). Dafür ist-soweit das Grundgesetz nichts anderes bestimmt-entsprechend Art. 42 Abs. 2 Satz 1 GG die Mehrheit der abgegebenen Stimmen erforderlich. Anhaltspunkte dafür, dass es je nach Höhe der Verpflichtungen oder Gewährleistungsübernahmen abgestufte Anforderungen an die parlamentarische Legitimation gäbe, sind nicht ersichtlich. Eine Erhöhung des Stammkapitals des Europäischen Stabilitätsmechanismus stellte weder eine Änderung des Grundgesetzes (vgl. Art. 79 Abs. 1 und Abs. 2 GG) noch eine Übertragung von Hoheitsrechten auf die Europäische Union dar, die das Grundgesetz seinem Inhalt nach ändern würde (vgl. Art. 23 Abs. 1 Satz 2 und Satz 3 iVm. Art. 79 Abs. 2 GG). "90

Diese Aussagen lassen sich ohne weiteres auf den Eigenmittelbeschluss übertragen: Ob das Stammkapital des ESM erhöht wird oder sich Deutschland zur zusätzlichen Finanzierung des europäischen Haushalts verpflichtet, macht unter wertenden Gesichtspunkten keinen Unterschied. In beiden Fällen verpflichtet man sich, Kapital zu einem nicht spezifizierten künftigen Zeitpunkt bereitzustellen. Für beide Fälle gilt solange es sich um Verpflichtungen in einem nicht wesentlichen Umfang handelt -

89 BVerfG 129, 124 Rn. 135 - EFSF.

90 BVerfG 135, 317 Rn. 388 - ESM-Vertrag. 
dass der Bundestag Herr seiner Entscheidungen bleibt und nicht fiskalischen Mechanismen ausgeliefert ist, die eine eigenständige Haushaltsbefassung unterminieren, in deren Rahmen der Bundestag fiskalische Verpflichtungen eingehen darf. Für diese reicht eine einfache Mehrheit aus. Das Bundesverfassungsgericht betont damit den Gleichlauf von Mehrheitserfordernissen in nationalen und supranationalen Sachverhalten.

\section{Wahrnehmung der Integrationsverantwortung}

Es versteht sich mittlerweile, dass es in Angelegenheiten der europäischen Integration nicht mit einer punktuellen Ratifikationsbefassung sein Bewenden hat. Dies ergibt sich insbesondere aus dem Mitwirkungsauftrag an das Parlament in Art. 23 Abs. 2 GG. Das Bundesverfassungsgericht hat hier den Begriff Integrationsverantwortung geprägt. ${ }^{91}$ Im vorliegenden Kontext genügen die bestehenden Unterrichtungspflichten der Bundesregierung nach dem Gesetz über die Zusammenarbeit von Bundesregierung und Deutschem Bundestag in Angelegenheiten der Europäischen Union (EUZBBG) bereits den verfassungsrechtlichen Anforderungen. Der Bundestag hat im März 2021 anlässlich der Ratifikation des Eigenmittelbeschlusses zur Ausfüllung dieser bereits bestehenden Verpflichtung im Konkreten weitere Vorgaben gemacht und festgelegt, dass die Bundesregierung, zeitnah und umfassend über den Entwicklungsstand des Aufbauinstruments „Next Generation EU“ zu berichten hat. ${ }^{92}$ Die Berichte sollen dem Deutschen Bundestag zusätzlich zu den bisherigen Unterrichtungen nach EUZBBG überblicksartig und jeweils ergänzt um eine Bewertung der Bundesregierung ein Gesamtbild der Einnahmen und Ausgaben des Aufbauinstruments, insbesondere der europäischen Kreditaufnahme, der geplanten und tatsächlichen Mittelverwendung der Aufbau- und Resilienzfazilität, den Entscheidungen im Wirtschafts- und Finanzausschuss bzw. im Europäischen Rat sowie den Sachstand bei den neuen Eigenmittel-Arten vermitteln. Der Bundestag sichert durch diese Betonung bestehender Verpflichtungen zusätzlich ab, dass das Parlament auf Grundlage der Berichte die zweckgemäße Verwendung der Mittel sachgerecht zu beurteilen vermag. Damit können auch frühzeitig mögliche Fehlentwicklungen identifiziert werden, die zu einer Heranziehung der Mitgliedstaaten führen könnten, um die Verpflichtungen der EU aus der Mittelaufnahme für NGEU nachzukommen. Das Parlament könnte dann mit der Bundesregierung solchen Entwicklungen entgegensteuern.

\section{Schlussbetrachtung}

Am Beispiel der Reform des Eigenmittelsystems lässt sich auf nationaler wie auf europäischer Ebene ein Demokratieparadox beobachten. Gesteigerte Anforderungen an Mehrheiten können ab einem bestimmten Punkt dazu führen, dass die Grundkonzeption von Demokratie als Mehrheitsherrschaft in den Hintergrund tritt: Sowohl das Einstimmigkeitserfordernis auf europäischer Ebene wie das Zweidrittelerfordernis auf

91 BVerfGE 123, 267 (355 f.) - Lissabon.

92 BT-Drs. 19/27921. 
nationaler Ebene kann dazu führen, dass eine Entscheidung von einigen wenigen abhängt, im äußersten Fall diese Minderheit der Mehrheit sogar sachfremde Bedingungen diktieren kann. Dies war der Fall für die Mitgliedstaaten Polen und Ungarn, die sich das Einstimmigkeitserfordernis im Rat für den Eigenmittelbeschluss zunutze machten, um sich an anderer Stelle Vorteile zu verschaffen. ${ }^{93}$ Auch bei der Sicherung einer Zweidrittelmehrheit in Bundestag und Bundesrat zeigt sich in einer immer unübersichtlicheren Parteienlandschaft, dass Mehrheiten von Kleinstparteien abhängen können.

Jedenfalls für die nationale Ebene in Deutschland spricht diese Beobachtung dafür, nicht leichtfertig das Erfordernis einer Zweidrittelmehrheit für Integrationsschritte anzunehmen. Das unter „Next Generation EU“ (NGEU) diskutierte Maßnahmenpaket hat aber sowohl aus europaverfassungsrechtlicher wie auch aus grundgesetzlicher Sicht auch nicht die Verfassungsdimensionen, die in der Vergangenheit bedeutende Verfassungsentwicklungen ausgelöst haben. Es geht nicht um die Einführung einer EU-Steuer, es besteht kein Anlass, den Schlachtruf der Boston Tea Party zu exhumieren - „,no taxation without representation“. Ein „Hamilton moment“ oder ein constitutional moment ${ }^{94}$ verbindet sich mit NGEU nicht. ${ }^{95}$ Es geht im Kern um ein inhaltlich wie zeitlich strikt zweckgebundenes und damit begrenztes Aufbauprogramm, das die verheerenden wirtschaftlichen Nebenfolge einer globalen Pandemie in der Europäischen Union abmildern helfen soll. Dafür stehen unionsrechtliche Rechtsgrundlagen zur Verfügung. Auch die Aufgeregtheit einiger Kritiker wegen einer angeblich neuen Verschuldungsmöglichkeit ist nüchtern betrachtet nicht begründet. Solange die EU weiter besteht, wird es auch einen EU-Haushalt und auf absehbare Zeit auch Beiträge der Mitgliedstaaten dazu geben. NGEU bedeutet letztlich nur, dass für einige Zeit ein fester Ausgabenposten für Schuldendienst im EU-Haushalt besteht, der jedenfalls durch Abstriche bei den EU-Ausgaben zu decken wäre. Dass dieses Maßnahmenpaket im geltenden Rechtsrahmen möglich ist, belegt ein weiteres Mal, dass das Potenzial der europäischen Verträge noch lange nicht ausgeschöpft ist, wenn politische Mehrheiten bestehen, um diese Potenziale zu aktivieren.

Gleichzeitig führt NGEU vor Augen, wie sehr die Union auch als wirtschaftspolitischer Akteur in Szene gesetzt werden kann und wie dies bereits in den Verträgen angelegt ist - siehe Art. 122 Abs. 1 AEUV. Das Narrativ, dass die Union hier lediglich auf Koordinierung und negative Integration im Sinne der Art. 123, 125 und 126 AEUV begrenzt ist, erweist sich immer mehr als überholt. Jedenfalls in der Krise soll und muss die Union wirtschaftspolitisch tätig werden, um die allgemeinen mitgliedstaatlichen Anstrengungen zu unterstützen und, gemäß ihrer Zielbestimmung aus Art. 3 EUV, „das Wohlergehen ihrer Völker zu fördern“.

93 Wegen der Sonderlage einer ratifikationsbedürftigen Sekundärrechtsetzung bestehen sogar zwei Ansatzmöglichkeiten für eine derartige Strategie: neben der Drohung im Rat die Einstimmigkeit zu vereiteln ist auch denkbar, dass Mitgliedstaaten mit einer Ablehnung der nationalen Ratifikation drohen, so wohl jüngst die baltischen Mitgliedstaaten, https://www.po litico.eu/article/baltics-threaten-to-hold-up-eu-coronavirus-recovery-fund-over-rail-project/.

94 S. dazu oben Fn. 54.

95 Entsprechend hat das BVerfG mit Beschl. v. 15.4.2021, 2 BvR 547/21, festgestellt, dass das Eigenmittelbeschluss-Ratifizierungsgesetz keine Zweidrittel-Mehrheit erforderte, schon weil es nicht einer Übertragung von Hoheitsrechten galt. 
Diesem Geiste entsprechend trägt die innerstaatliche Zustimmungsgesetzgebung der rechtlichen Überschaubarkeit der vorgeschlagenen Maßnahmen mit entsprechendem Augenmaß Rechnung. Der durch das deutsche Verfassungsrecht gezogene und bereits in mit verfassungsändernden Mehrheiten - zuletzt zum Vertrag von Lissabon konkretisierte Rechtsrahmen ist bei weitem noch nicht ausgeschöpft. 